\title{
Coexistence of giant Cooper pairs with a bosonic condensate and anomalous behavior of energy gaps in the BCS-BEC crossover of a two-band superfluid Fermi gas
}

\author{
Yuriy Yerin $\odot,{ }^{1}$ Hiroyuki Tajima $\odot,{ }^{2}$ Pierbiagio Pieri $\odot,{ }^{1,3}$ and Andrea Perali ${ }^{4}$ \\ ${ }^{1}$ School of Science and Technology, Physics Division, Università di Camerino, 62032 Camerino (MC), Italy \\ ${ }^{2}$ Quantum Hadron Physics Laboratory, RIKEN Nishina Center, Wako, Saitama, 351-0198, Japan \\ ${ }^{3}$ INFN, Sezione di Perugia, 06123 Perugia (PG), Italy \\ ${ }^{4}$ School of Pharmacy, Physics Unit, Università di Camerino, 62032 Camerino (MC), Italy
}

(Received 30 July 2019; revised manuscript received 19 September 2019; published 30 September 2019)

\begin{abstract}
We investigate the Bardeen-Cooper-Schrieffer-Bose-Einstein condensation crossover in a two-band superfluid Fermi gas with an energy shift between the bands. When the intraband coupling in the cold (first) band is fixed as weak, we find that in the case of vanishing pair-exchange interband coupling and in the strong-coupling limit of the hot (second) band the system undergoes a transition to a single-component configuration with a full suppression of the first energy gap and a full redistribution of particles between bands. For nonvanishing pair-exchange interband coupling we reveal a nonmonotonic dependence of the energy gap in the first band vs intraband coupling in the second band, with the presence of a hump. In the case of weak interband coupling, the system shows a significant amplification of the intrapair correlation length of the condensate in the first band in the strong-coupling regime of the second band, which clearly indicates the coexistence of giant Cooper pairs and a bosonic condensate even for nonzero temperatures. This can lead to a nonmonotonic temperature dependence of the second energy gap with a peak. The here predicted coexistence of giant Cooper pairs and bosonic molecules can be verified by means of the visualization of vortex cores in two-component atomic condensates as well as in some iron-based superconductors.
\end{abstract}

DOI: 10.1103/PhysRevB.100.104528

\section{INTRODUCTION}

A model of two-band superfluidity has been considered for a long time solely as the next iteration step to the BardeenCooper-Schrieffer (BCS) theory of superconducting state to take into account the anisotropic properties of metals and the effect of overlapping of the energy bands in the vicinity of their Fermi surface, which leads to the appearance of interband quantum electron transitions and, as a result, to an additional indirect interaction between the electrons of each band $[1,2]$. The explosive growth in the study of multiband superconductivity began from the discovery of unconventional superconductivity with a complex structure of the superconducting order parameter (cuprates, heavy-fermion compounds, borocarbides, fullerides, strontium ruthenate, organic superconductors, iron pnictides, and chalcogenides). The complex structure of the order parameter gives rise to a much richer nomenclature of topological objects and effects in unconventional superconductors in comparison with their conventional counterparts. These superconducting systems can lead to the formation of a variety of quantum phenomena: states that break time-reversal symmetry (BTRS), new collective modes, phase domains, vortices with fractional flux, fractional Josephson effect [3-6], and shape resonance in the superconducting properties $[7,8]$.

Another intriguing aspect is that compounds with unconventional superconductivity can demonstrate anomalous normal-state properties above their critical temperature, which are interpreted as the pseudogap state. The existence of a pseudogap state has been first argued in the context of the crossover from BCS superconductivity to Bose-Einstein condensation (BEC) in the ground state and at finite temperature $[9,10]$ for underdoped high- $T_{c}$ cuprate superconductors [11-13]. In these compounds, pseudogap formation and non-Fermi-liquid behavior are well established, and unusual superconducting fluctuations have also been detected above the critical temperature. However, the pseudogap state appears at a much higher temperature than the onset temperature of superconducting fluctuations. At this moment, it is still a debated question whether the system is deep inside the crossover regime and to what extent the crossover physics can be relevant to the phase diagram of underdoped cuprate superconductors.

A magnesium diboride superconductor $[14,15]$ and the recently discovered family of iron-based superconductors with the multiband electron structure and multiple energy gaps offer a new platform for the experimental observation of the BCS-BEC crossover, providing an opportunity to study new problems about crossover, fluctuation phenomena, and pseudogap in multicomponent systems, which go beyond the single-band physics [16]. For instance, $\mathrm{BaFe}_{2}\left(\mathrm{As}_{1-\mathrm{x}} \mathrm{P}_{\mathrm{x}}\right)_{2}$ may approach the BCS-BEC crossover regime near a quantum critical point $[17,18]$. Another candidate is iron chalcogenide $\mathrm{Fe}_{1+\mathrm{y}} \mathrm{Se}_{\mathrm{x}} \mathrm{Te}_{1-\mathrm{x}}$, in which the Fermi energy of FeSe is extremely small and can be tuned by chemically doping through the BCS-BEC crossover [19-22]. It was found experimentally that the dimensionless measure of the pairing strength, i.e., the ratio between the energy gap and the Fermi energy 
$\Delta / E_{F}=0.16,0.3$, and 0.5 , increases monotonically with decreasing of the iron excess $y$, exhibiting a crossover from the $\mathrm{BCS}$ to the BEC regime [23]. The investigation of the vortex core by means of scanning tunneling microscopy (STM) shows the presence of Friedel-type oscillations, confirming the BCS-BEC crossover nature of FeSe and a peculiar missing of the pseudogap [24].

Despite that for most of multiband superconducting systems the tuning of pair-exchange interband or intraband interactions is rather challenging and their properties can not be studied easily away from the BCS regime. Strongly interacting superfluid systems can be replicated experimentally with ultracold atomic Fermi gases in optical lattices or in single traps confining clouds of fermionic atoms with several hyperfine states [25-27]. In such systems the interaction strength is adjusted by means of Fano-Feshbach resonances which allow the evolution of superfluidity throughout the BCS-BEC crossover. The newly realized orbital Feshbach resonance in a ${ }^{173} \mathrm{Yb}$ Fermi gas promises a new way for studying two-band Fermi system with Josephson-type pair-exchange interaction between bands, enabling the tuning of interorbital interactions based on the Zeeman shift of different nuclear spin states of the atoms [28-30]. The many-body Hamiltonian governing the physical properties of alkaline-earth-metal Fermi gases across an orbital Feshbach resonance is similar to that of twoband $s$-wave superconductors, and the description of the BCS$\mathrm{BEC}$ crossover in these systems requires two components of the order parameter, in contrast to a Fermi gas with a single orbital near a broad magnetic Feshbach resonance. Thus, experimental activity in this direction raises fundamentally new problems about the BCS-BEC crossover in multiband superfluids and calls for the theoretical predictions of possible unusual effects [31-40]. At this moment, the evolution of low-energy collective excitations from BCS to BEC coupling regime in two-band $s$-wave superfluids coupled via an interband Josephson interaction at $T=0$ has been studied [31]. Later within a mean field theory generalized to the case of two bands, the characteristics of two-band superfluidity throughout the BCS-BEC crossover were analyzed and results have been reported only for coincident bands [32]. Furthermore, based on the extension of the Nozières-Schmitt-Rink approach [9] for two bands, a strong enhancement of the critical temperature, a significant reduction of the preformed pair region where pseudogap effects are expected, and the entanglement of two kinds of composite bosons in the strongcoupling BEC regime were predicted for a two-band attractive Fermi system in the normal state with a shallow band coupled to a weakly interacting deeper band [40].

In this paper, using a mean field theory for a two-band superfluid with gap equations coupled to the density equation we show that a two-band superfluid Fermi gas with energy shift between the bands reveals unique features of the BCS-BEC crossover, which are not realized in the single-band system. The paper is organized as follows. In Sec. II, we present the model and the main equations of a mean field approach for the description of the BCS-BEC crossover in a two-band system. In Sec. III, we provide the results of our numerical calculations for the energy gaps, chemical potential, particle densities, and the intrapair correlation lengths and discuss unique features of the BCS-BEC crossover, in particular, the

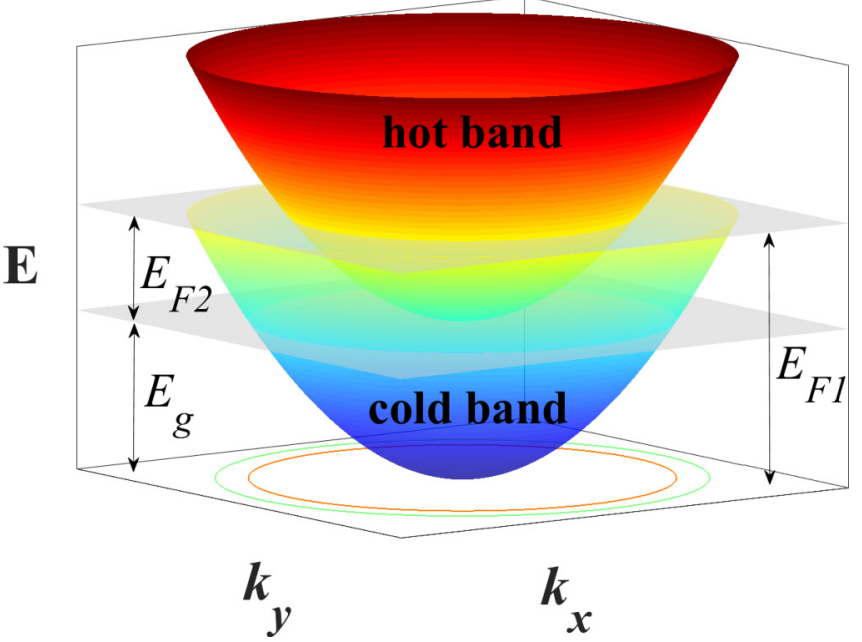

FIG. 1. The band structure of the two-band superfluid Fermi gas under consideration ( $k_{z}=0$ projection). $E_{\mathrm{g}}$ is the energy shift between the first $(i=1)$ and the second $(i=2)$ band. $E_{\mathrm{F} i}$ corresponds to the Fermi energy of $i$ band in the absence of interactions.

coexistence of giant Cooper pairs and bosonic condensate in the strong-coupling regime. We summarize our conclusions in Sec. IV. Two appendices with analytical calculations and technical details are reported at the end of the paper.

\section{MODEL AND BASIC EQUATIONS}

We consider a two-band system of interacting fermions in three dimensions (3D), where the two fermionic bands have a parabolic dispersion law

$$
\xi_{i}(\mathbf{k})=\frac{|\mathbf{k}|^{2}}{2 m}-\mu+\epsilon_{i},
$$

where $\mathbf{k}$ is the wave vector, $m$ the effective mass which is assumed equal for both bands, $\mu$ the chemical potential, and $\epsilon_{i}$ the energy of the bottom of the bands. The index $i=1,2$ numerates the bands, where $i=1$ denotes the lower band and $i=2$ is the upper band. We set $\epsilon_{1}=0$ and $\epsilon_{2}=E_{\mathrm{g}}$ where the value $E_{\mathrm{g}}$ defines the energy shift between the two bands of the system (Fig. 1).

The effective interaction between fermions is approximated by a separable potential

$$
V_{i j}\left(\mathbf{k}, \mathbf{k}^{\prime}\right)=-U_{i j} \Theta\left(k_{0}-|\mathbf{k}|\right) \Theta\left(k_{0}-\left|\mathbf{k}^{\prime}\right|\right),
$$

where $U_{i j}$ are the strength of intraband (when $i=j$ ) and pair-exchange interband (when $i \neq j$ ) interactions, $k_{0}$ is the cutoff momentum, which is supposed to be the same for intraband and interband terms, and $\Theta(x)$ is the Heaviside function. The sign of $U_{12}$ determines the symmetry of the order parameter in the clean case. A repulsive pair-exchange interband interaction $U_{12}<0$ leads to a ground state with $\pi$-phase difference between the two bands, while attractive interband interactions $U_{12}>0$ stabilize a ground state with a zero-phase difference between their gap functions [41].

The ground state of the two-band system is examined within a mean field theory. We generalize the single-band approach to the two-band case and write the equations for the 
energy gaps

$$
\Delta_{i}(\mathbf{k})=-\frac{1}{\Omega} \sum_{j} \sum_{k^{\prime}} V_{i j}\left(\mathbf{k}, \mathbf{k}^{\prime}\right) \frac{\Delta_{j}\left(\mathbf{k}^{\prime}\right) \tanh \frac{E_{i}\left(\mathbf{k}^{\prime}\right)}{2 T}}{2 E_{i}\left(\mathbf{k}^{\prime}\right)} .
$$

Here, $\Omega$ is the volume occupied by the system under consideration, $E_{i}\left(\mathbf{k}^{\prime}\right)=\sqrt{\xi_{j}^{2}\left(\mathbf{k}^{\prime}\right)+\Delta_{j}^{2}\left(\mathbf{k}^{\prime}\right)}$ are excitation branches in the superfluid state, and the gaps have the same cutoff generated by the separable interaction

$$
\Delta_{i}(\mathbf{k})=\Delta_{i} \Theta\left(k_{0}-|\mathbf{k}|\right) .
$$

The coupled equations for the energy gaps must be supplemented with the equation for the total particle density of the system, as the renormalization of the chemical potential is a key feature of the BCS-BEC crossover. We consider the total density of particles of the two-band system in the form of an additive contribution from each band

$$
n=n_{1}+n_{2},
$$

where $n_{i}$ is the particle density in each band

$$
n_{i}=\frac{2}{\Omega} \sum_{\mathbf{k}}\left[v_{i}^{2}(\mathbf{k}) f\left(-E_{i}(\mathbf{k})\right)+u_{i}^{2}(\mathbf{k}) f\left(E_{i}(\mathbf{k})\right)\right],
$$

where $f(z)$ is the Fermi-Dirac distribution function and the weights $v_{i}(\mathbf{k})$ and $u_{i}(\mathbf{k})$ are defined by

$$
\begin{gathered}
v_{i}^{2}(\mathbf{k})=\frac{1}{2}\left[1-\frac{\xi_{i}(\mathbf{k})}{E_{i}(\mathbf{k})}\right], \\
u_{i}^{2}(\mathbf{k})=1-v_{i}^{2}(\mathbf{k}) .
\end{gathered}
$$

During the calculations, $n$ will be taken constant $n=n_{1}^{0}+n_{2}^{0}=\frac{k_{\mathrm{F} 1}^{3}}{3 \pi^{2}}+\frac{k_{\mathrm{F} 2}^{3}}{3 \pi^{2}}=\frac{k_{\mathrm{Ft}}^{3}}{3 \pi^{2}}$, where we have defined the particle densities $n_{i}^{0}$ in the absence of interactions and at zero temperature, as well as the corresponding Fermi momentum for each band $k_{\mathrm{F} i}$ and total Fermi momentum $k_{\mathrm{Ft}}$. According to the model of the two-band system we also assume the presence of an energy shift between bands $E_{\mathrm{g}}=\eta E_{\mathrm{F} 2}$, where $E_{\mathrm{F} 2}=k_{\mathrm{F} 2}^{2} / 2 m$ (and we assumed, as in Fig. 1, $E_{\mathrm{g}}<E_{\mathrm{F} 1}$, such that the upper band is partially occupied in the noninteracting case at zero temperature). This implies the relations between different Fermi momenta $k_{\mathrm{F} 1}=\left[1-\frac{1}{(\eta+1)^{\frac{3}{2}}+1}\right]^{\frac{1}{3}} k_{\mathrm{Ft}}$ and $k_{\mathrm{F} 2}=\left[\frac{1}{(\eta+1)^{\frac{3}{2}}+1}\right]^{\frac{1}{3}} k_{\mathrm{Ft}}$. For regularization we use the $s$-wave scattering lengths for each band $a_{i i}$ defined by the low-energy limit of the two-body problem in vacuum

$$
\frac{m}{4 \pi a_{i i}}=-\frac{1}{U_{i i}}+\sum_{\mathbf{k}}^{\mathbf{k}_{0}} \frac{m}{\mathbf{k}^{2}},
$$

where the momentum cutoff $k_{0}$ is much larger than the inverse of the average distance between particles, namely, $k_{0} \gg k_{\mathrm{Ft}}$. We will show that in this way the selection of the momentum cutoff will not affect the obtained results (see Appendices A and B). For the sake of simplification we redefine constants $U_{i j}=\tilde{U}_{i j}\left(\frac{k_{\mathrm{Ft}}}{k_{0}}\right)^{2} \frac{E_{\mathrm{Ft}}}{n}$ of the intraband $(i=j)$ and the interband $(i \neq j)$ exchange couplings, where $E_{\mathrm{Ft}}=k_{\mathrm{Ft}}^{2} / 2 m$ is the total Fermi energy. From Eq. (9) this yields relations in the dimensionless form between intraband coupling coefficients and scattering lengths for each band

$$
\tilde{U}_{i i}\left(\frac{k_{\mathrm{Ft}}}{k_{0}}\right)^{2}=\frac{4}{3}\left(\frac{k_{0}}{k_{\mathrm{Ft}}}-\frac{\pi}{2 k_{F i} a_{i i}} \frac{k_{F i}}{k_{\mathrm{Ft}}}\right)^{-1} .
$$

Substituting Eq. (10) into Eq. (3) and measuring momenta in units of $k_{\mathrm{Ft}}$ and energies in units of $E_{\mathrm{Ft}}$ in Eqs. (3) and (6), we get the system of dimensionless equations for the energy gaps and the particle densities that will be solved numerically (see Appendix A).

Aside from the energy gaps $\Delta_{i}$ and the particle densities $n_{i}$, another important characteristic of the pairing regimes throughout the BCS-BEC crossover in a two-band superfluid Fermi gas is the intrapair correlation lengths of the Cooper pairs, which is determined by the expression

$$
\xi_{\text {pair }, i}^{2}=\frac{\sum_{\mathbf{k}}\left|\nabla_{\mathbf{k}}\left(\frac{1-2 f\left(E_{i}(\mathbf{k})\right)}{E_{i}(\mathbf{k})}\right)\right|^{2}}{\sum_{\mathbf{k}}\left(\frac{1-2 f\left(E_{i}(\mathbf{k})\right)}{E_{i}(\mathbf{k})}\right)^{2}},
$$

obtained from the pair correlation function, evaluated at a mean field level for zero and finite temperature [42].

Differently from the paper [32] where the ratio of intraband coupling constants was fixed for the investigation of the BCSBEC crossover properties, we study a two-band system with a fixed value of scattering length for the first band, which corresponds to the $\mathrm{BCS}$ regime, namely, $\left(k_{\mathrm{F} 1} a_{11}\right)^{-1}=-2$, and a varying scattering length for the second band $\left(k_{\mathrm{F} 2} a_{22}\right)^{-1}$. The use of the scattering lengths as physical parameters, which are kept fixed when the momentum cutoff $k_{0}$ is varied, avoids any convergence problem and the dependence of physical quantities on the momentum cutoff $k_{0}$ when $k_{0}$ is sufficiently large (see Appendix B).

We fix the energy shift between bands $E_{\mathrm{g}}=0.75 E_{\mathrm{F} 1}=$ $3 E_{\mathrm{F} 2}$, with corresponding relations between the Fermi momenta in each band and the total Fermi momentum: $k_{\mathrm{F} 1}=$ $(8 / 9)^{1 / 3} k_{\mathrm{Ft}}$ and $k_{\mathrm{F} 2}=(1 / 9)^{1 / 3} k_{\mathrm{Ft}}$. This choice of the energy shift is representative of the situation of most physical interest with $0<E_{\mathrm{g}}<E_{\mathrm{F} 1}$, in which both bands are partially occupied in the absence of interactions at zero temperature. When instead $E_{\mathrm{g}}>E_{\mathrm{F} 1}$, the upper band is empty and all particles reside in the lower band in the absence of interaction. In particular, in the large energy shift limit $E_{\mathrm{g}} \gg E_{\mathrm{F} 1}$, the upper band matters only if the intraband interaction in the second band is made so strong that the energy of the two-body bound state forming in this band approaches the Fermi level of the first band. This is actually the physical mechanism of a Feshbach resonance, with the interband coupling controlling the width of the resonance. In this limit, the two-band model thus effectively reduces to the so-called two-channel model, widely studied in the context of ultracold gases [43-45]: in this situation the continuum of the second band becomes irrelevant and only the bound-state (boson) forming from the second band needs to be considered. 

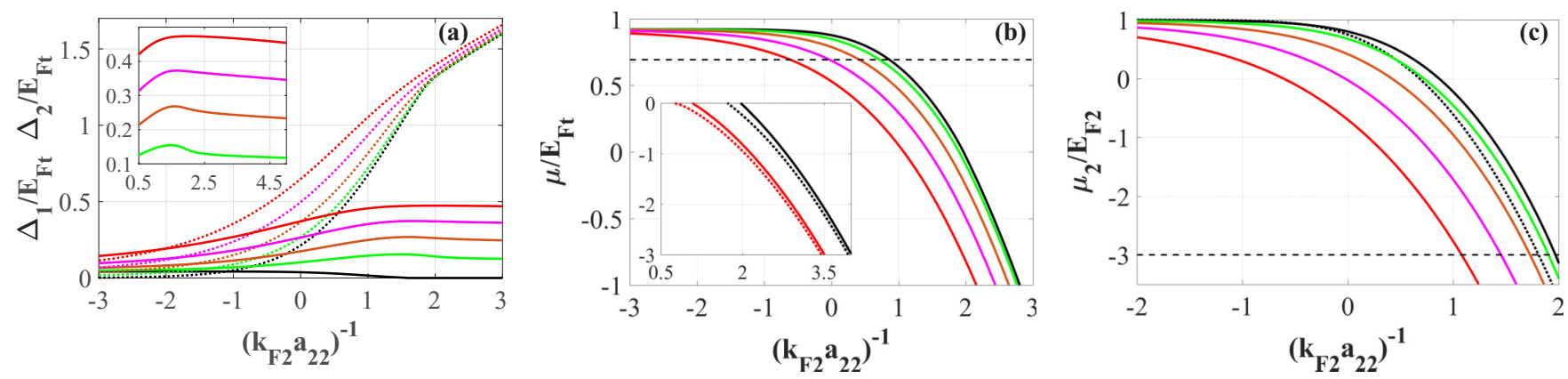

FIG. 2. (a) Energy gaps $\Delta_{1}$ (solid lines), $\Delta_{2}$ (dotted lines), (b) the chemical potential $\mu$, and (c) the chemical potential $\mu_{2}=\mu-E_{\mathrm{g}}$ in units of $E_{\mathrm{F} 2}$ at zero temperature as a function of $\left(k_{\mathrm{F} 2} a_{22}\right)^{-1}$ for different interband couplings $\tilde{U}_{12}=0$ (black line), $\tilde{U}_{12}=0.5$ (green line), $\tilde{U}_{12}=1$ (brown line), $\tilde{U}_{12}=1.5$ (magenta line), $\tilde{U}_{12}=2$ (red line) with the fixed value of the scattering length in the first band $\left(k_{\mathrm{F} 1} a_{11}\right)^{-1}=-2$. The presence of the hump on energy gap $\Delta_{1}$ dependencies for different interband interaction strengths is shown in the inset. Dashed black line in (b) and (c) corresponds to the energy shift $E_{\mathrm{g}}$ between bands in units of $E_{\mathrm{Ft}}$ and $E_{\mathrm{F} 2}$, respectively. Dotted black line in (c) is the chemical potential of a single-band superfluid Fermi gas. Inset in (b) shows the comparison between the chemical potential (solid lines) and half of the binding energy $-E_{b} / 2$ (dotted lines) dependencies in the strong-coupling limit.

\section{RESULTS AND DISCUSSION}

\section{A. Energy gaps, chemical potential, and particle densities}

To provide a comprehensive description of the BCS-BEC crossover properties in a two-band superfluid Fermi gas, first of all we analyze the evolution of the energy gaps, the chemical potential, and the particle densities at zero temperature based on the numerical solution of Eqs. (3)-(6). It should be noted that in principle for $T=0$ the system of Eqs. (3)-(6) can be integrated analytically and after long but straightforward calculations it is expressed via full elliptic integrals of the first and the second kinds. These analytical calculations show that, at least for zero temperature, within our strategy with fixed scattering lengths there is no dependence on the cutoff $k_{0}$ for $\Delta_{i}$ and $\mu$ for $k_{0} \gg k_{\mathrm{Ft}}$. The same statement can be extended analytically for the case of $T_{c}$ (see Appendix A).

We found that in a system with vanishing interband interaction, the BCS-BEC crossover is characterized by the full suppression of the first energy gap in the BEC limit and the presence of a kink on the second gap dependence at $\left(k_{\mathrm{F} 2} a_{22}\right)^{-1} \approx 2$ [Fig. 2(a)]. The interband coupling smooths out the kink of the second gap and leads to the activation of the first gap in the BEC limit. Despite that Fig. 2(a) shows the almost constant character of $\Delta_{1}$ dependence for the interval $\left(k_{\mathrm{F} 2} a_{22}\right)^{-1} \in[-3 ; 3]$, according to our numerical analysis we observe a very slow increase of the first gap starting from the nonzero value of $\Delta_{1}^{(0)} \approx 0.043$ in the BCS limit and a very slow decrease of $\Delta_{1}\left(1 / k_{\mathrm{F} 2} a_{22}\right)$ in the BEC limit. Moreover, the behavior of $\Delta_{1}\left(1 / k_{\mathrm{F} 2} a_{22}\right)$ has always a nonmonotonic dependence, with a very tiny hump in the BCS limit in the case of vanishing interband interaction. With the further increasing of the interband coupling, this hump becomes more pronounced and is shifted to the BEC limit [see inset in Fig. 2(a)]. Note that for weak interband coupling the energy gap in the first band is exponentially suppressed when the cold band is almost depleted. The overall nonmonotonic behavior of $\Delta_{1}$ as a function of $\left(k_{\mathrm{F} 2} a_{22}\right)^{-1}$ indicates a first regime of weak to intermediate coupling in the hot band in which $\Delta_{1}$ increases because of the effective attraction generated by the interband interaction, which is able to transfer attractive pairing from the hot to the cold band. On the other hand, when the coupling in the hot band becomes very strong, the depletion of the cold band starts to dominate, causing a decrease in $\Delta_{1}$ and the presence of the hump in $\Delta_{1}$ is the result of this interplay.

The chemical potential of a two-band superfluid Fermi gas decreases, in turn, slower in comparison with the single-band counterpart even in the presence of the interband interaction [Fig. 2(b)]. It is important to note that the single-band case differs from the two-band one for $U_{12}=0$ because a particle transfer between the two bands occurs due to the additive structure of the density equation in Eq. (6).

Based on the two-body Schrödinger equation we calculate the dependence of the two-body binding energy $E_{b}$ for different interband couplings [inset in Fig. 2(b)]. One can see that when $U_{12}$ increases, $E_{b}$ also increases, and the chemical potential of the system tends to the BEC limit $-E_{b} / 2$.

In Fig. 2(c) we report the behavior of $\mu_{2} \equiv \mu-E_{\mathrm{g}}$ normalized to $E_{\mathrm{F} 2}$ as a function of $\left(k_{\mathrm{F} 2} a_{22}\right)^{-1}$ to compare with the single-band result. For vanishing or weak interband coupling $\left(\tilde{U}_{12}<1\right)$, the chemical potential can be larger than for a single-band case. This indicates Pauli-blocking effects due to the cold states as already obtained in the vicinity of the critical temperature by a Nozières-Schmitt-Rink approach in Ref. [40].

For $\tilde{U}_{12}=\tilde{U}_{21}=0$ the full suppression of $\Delta_{1}$ is connected with the full redistribution of particles between bands (Fig. 3) Even though the density equation (6) couples two condensates, the first band remains in the BCS regime and until unitarity the particle distribution among the two bands is not important for the system. Increasing the interband interaction favors an equal distribution of particles in the weak-coupling regime and a retardation of the redistribution process between bands in the strong-coupling limit.

Until now we have investigated the characteristics of the BCS-BEC crossover in a two-band fermionic system with the fixed value $\left(k_{\mathrm{F} 1} a_{11}\right)^{-1}=-2$ of intraband coupling in the first band. To understand full properties of the BCSBEC crossover for this system at $T=0$, we consider the behavior of the energy gaps and of the particle densities by 


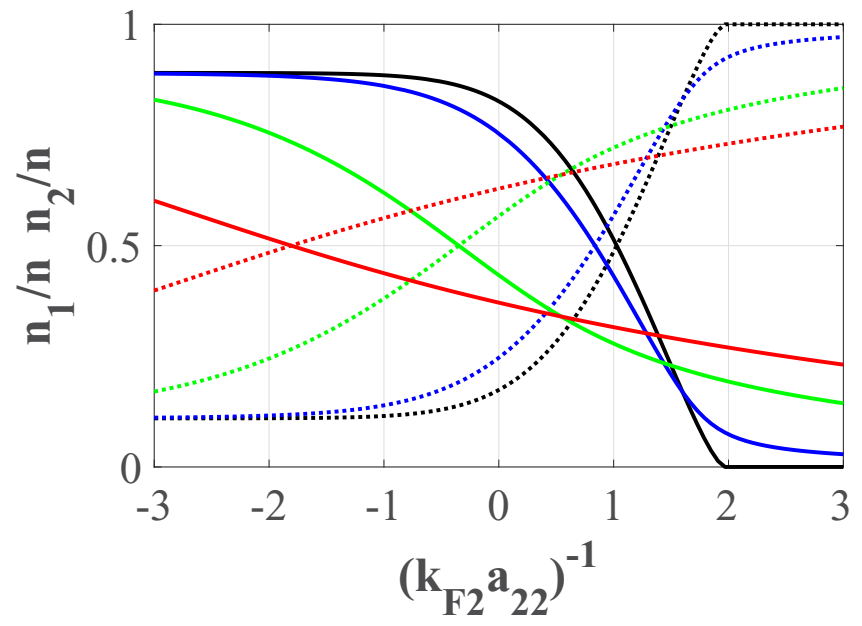

FIG. 3. Distribution of particle densities in each band $n_{1} / n$ (solid lines) and $n_{2} / n$ (dotted lines) normalized to the total particle densities as a function of $\left(k_{\mathrm{F} 2} a_{22}\right)^{-1}$ for different interband couplings $\tilde{U}_{12}=0$ (black line), $\tilde{U}_{12}=1$ (blue line), $\tilde{U}_{12}=3$ (green line), and $\tilde{U}_{12}=5$ (red line) with $\left(k_{\mathrm{F} 1} a_{11}\right)^{-1}=-2$ and at $T=0$.

varying $\left(k_{\mathrm{F} 1} a_{11}\right)^{-1}$. First of all, as we can see from Figs. 4(a) and 4(b), a weak intraband coupling in the first band and a strong in the second one, namely, when $\left(k_{\mathrm{F} 1} a_{11}\right)^{-1}<0$ and $\left(k_{\mathrm{F} 2} a_{22}\right)^{-1}>2$ together with a vanishing interband interaction transform the two-band system into the single-band one with the first gap fully suppressed. The single-band scenario is realized also for $\left(k_{\mathrm{F} 1} a_{11}\right)^{-1}>0$ and for the entire interval of values $\left(k_{\mathrm{F} 2} a_{22}\right)^{-1}$ with the full suppression of the second gap. Increasing the interband interaction extends the region and broadens the borders, where the two-band configuration is preserved [Figs. 4(c) and 4(d)].

It is worth noting that a similar transition from singlecondensate to two-condensate superconductivity was revealed experimentally in the $\mathrm{LaAlO}_{3} / \mathrm{SrTiO}_{3}$ interface driven by electrostatic doping [46]. It was found that in such a
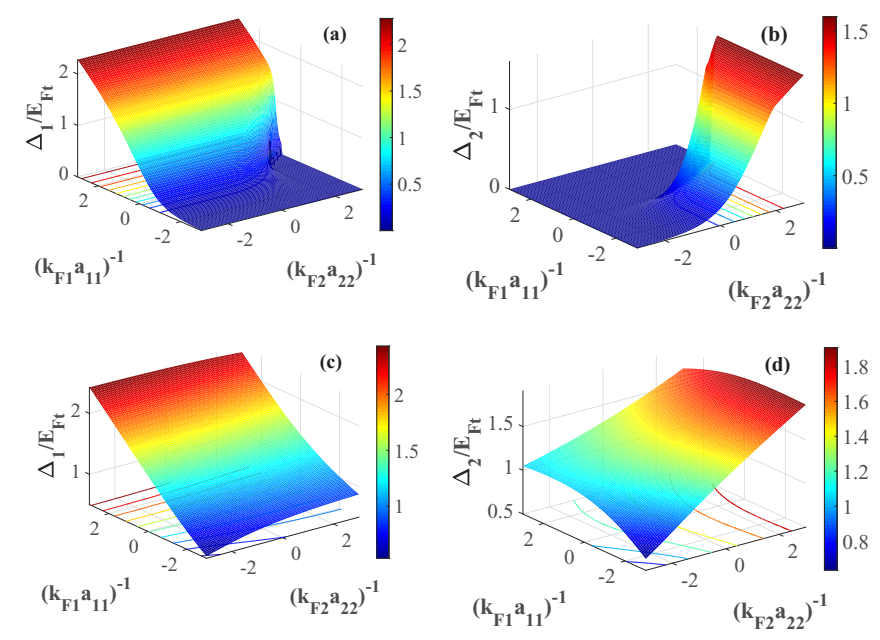

FIG. 4. Evolution of energy gaps $\Delta_{1}$ (a), (c) and $\Delta_{2}$ (b), (d) at zero temperature vs scattering lengths for fermions in the first and second bands for different interband couplings: $\tilde{U}_{12}=0$ (a), (b) and strongly coupled bands $\tilde{U}_{12}=5$ (c), (d).
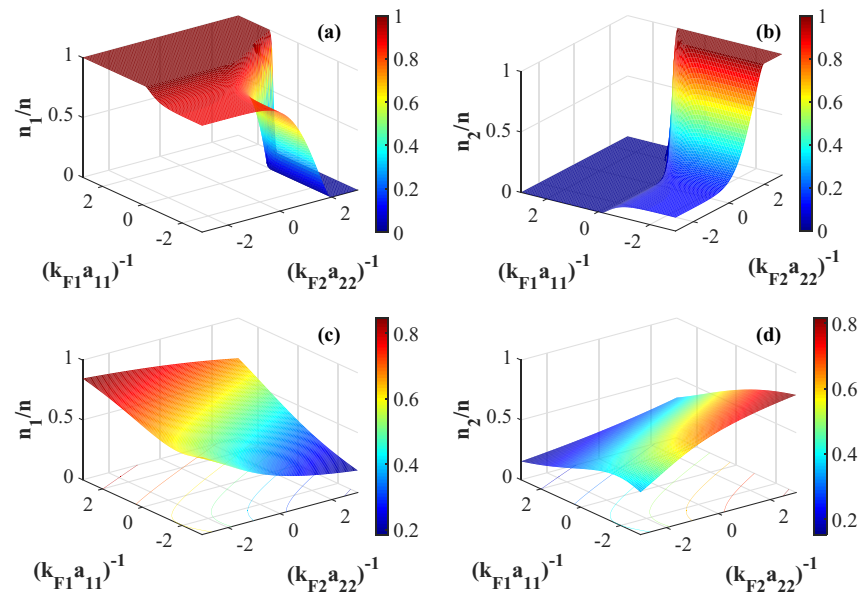

FIG. 5. Normalized particle densities in the first (a), (c) and in the second band (b), (d) as a function of $\left(k_{\mathrm{F} 1} a_{11}\right)^{-1}$ and $\left(k_{\mathrm{F} 2} a_{22}\right)^{-1}$ for different values of interband interaction strengths $\tilde{U}_{12}=0$ (a), (b) and $\tilde{U}_{12}=5$ (c), (d) at $T=0$.

heterostructure the superconducting gap in the first band is suppressed while the second band is populated. Within our approach, we speculate that these results can be interpreted as the transition from the BEC to the BCS regime of a two-band superfluid system close to a Lifshitz transition with vanishing interband interaction. Even though the heterostructures $\mathrm{LaAlO}_{3} / \mathrm{SrTiO}_{3}$ represent the two-dimensional electron liquid in the interface, some theoretical models argue the importance of the three-dimensional bands for the explanation of 2D superconductivity in these systems [47].

Our results are confirmed also by the evolution of the particle densities, whereby, as it can be seen from Figs. 5(a) and $5(b)$, there is no transfer of particles for vanishing interband interaction for $\left(k_{\mathrm{F} 1} a_{11}\right)^{-1}>0$ with all particles concentrated in the deeper band, while in the opposite case for $\left(k_{\mathrm{F} 1} a_{11}\right)^{-1}<0$ and $\left(k_{\mathrm{F} 2} a_{22}\right)^{-1}>2$ the bands change places: all particles migrate to the shallow band. Figures 5(c) and 5(d) show that when the interband coupling increases, the populations in each band tend to equalize.

\section{B. Intrapair correlation lengths}

Another characteristic for the description of the crossover from Cooper-pair superconductivity to Bose-Einstein condensation of bound pairs of fermions is the intrapair correlation length that is defined by Eq. (11). For a single-band system it was shown earlier that there is a universal materialindependent criterion based on $k_{F} \xi$ to follow the evolution of the BCS-BEC crossover [48]. Based on Eq. (11) and the definition from the paper [48], we investigate the intrapair correlation lengths for each band $\xi_{\text {pair } 1}$ and $\xi_{\text {pair2 }}$ as a function of the scattering length in the second band $\left(k_{\mathrm{F} 2} a_{22}\right)^{-1}$.

In the case of vanishing interband interaction $\xi_{\text {pair } 2}$ has a conventional behavior as in the single-band case, whereas $\xi_{\text {pairl }}$ is almost constant until the unitarity point and undergoes an essential discontinuity (divergence) at $\left(k_{\mathrm{F} 2} a_{22}\right)^{-1} \approx 2$ [Fig. 6(a)]. The origin of this divergence can be understood from the definition of $\xi_{\text {pairl }}$ after straightforward integration of Eq. (11). The obtained expression diverges when 

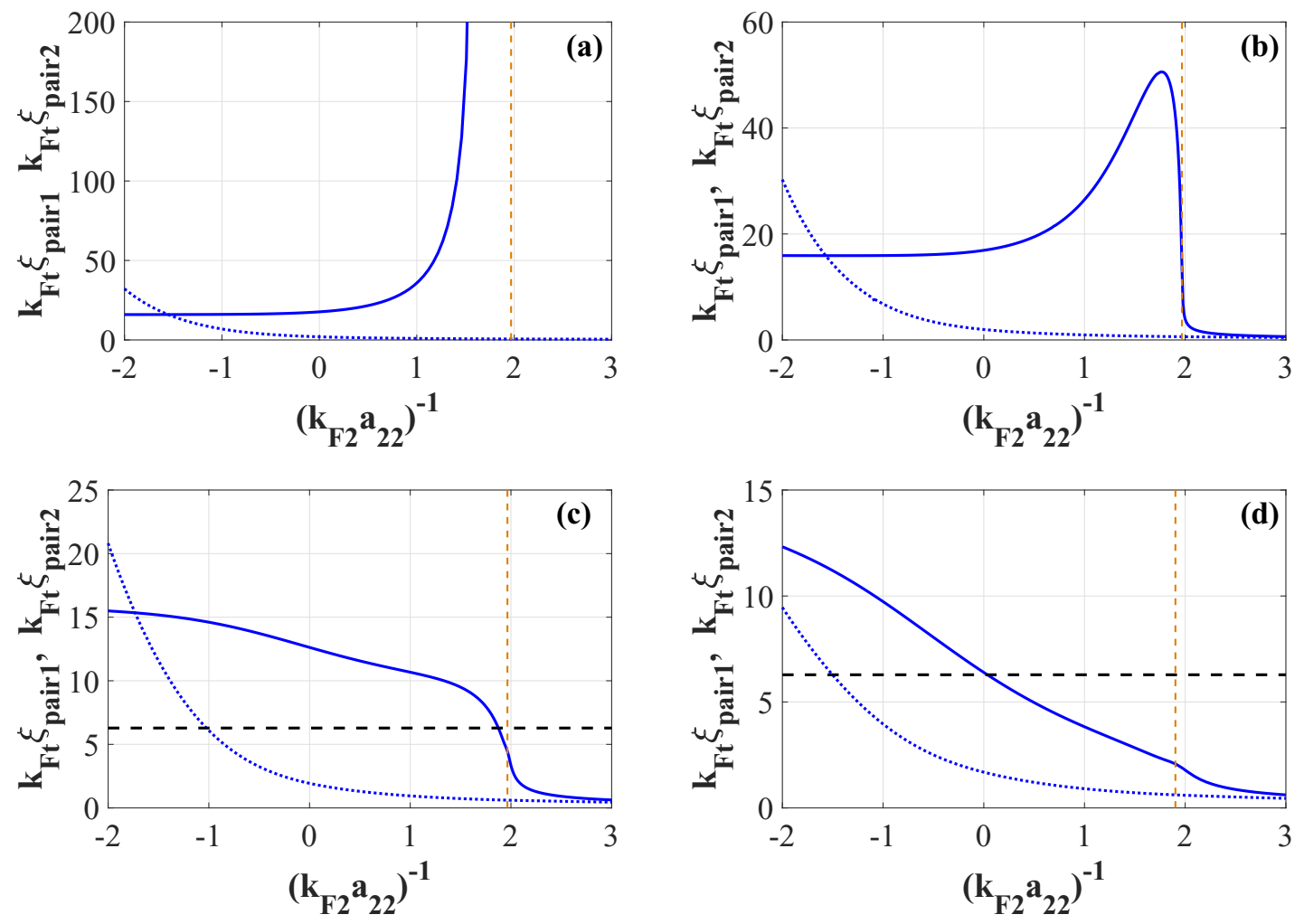

FIG. 6. Intrapair correlation lengths $\xi_{\text {pair1 }}$ and $\xi_{\text {pair2 }}$ for the first (solid lines) and the second band (dotted lines) correspondingly at zero temperature as a function of $\left(k_{\mathrm{F} 2} a_{22}\right)^{-1}$ for different interband couplings strengths $\tilde{U}_{12}=0$ (a), $\tilde{U}_{12}=0.01$ (b), $\tilde{U}_{12}=0.1$ (c), and $\tilde{U}_{12}=0.5$ (d) in the case $\left(k_{\mathrm{F} 1} a_{11}\right)^{-1}=-2$. Dashed black lines in (c) and (d) correspond to $k_{F} \xi_{\text {pair }}=2 \pi$ and delimit the BCS-BEC crossover regime [due to large values of $\xi_{\text {pair } 1}$ and $\xi_{\text {pair } 2}$ in panels (a) and (b) we did not plot $k_{F} \xi_{\text {pair }}=2 \pi$ ]. Orange dashed lines define the value of the coupling strength in the second band for which the chemical potential of the system equals to zero [see Fig. 2(b)], namely, $\left(k_{\mathrm{F} 2} a_{22}\right)^{-1} \approx 1.968$ in (a) and (b), $\left(k_{\mathrm{F} 2} a_{22}\right)^{-1} \approx 1.965$ in $(\mathrm{c})$, and $\left(k_{\mathrm{F} 2} a_{22}\right)^{-1} \approx 1.903$.

the energy gap in the first band $\Delta_{1}=0$ is fully suppressed [Fig. 2(a)].

By increasing the interband coupling, this divergence is removed. For very weak interaction between bands, a sharp peak on the dependence of $\xi_{\text {pair } 1}$ on $1 / k_{\mathrm{F} 2} a_{22}$ is obtained [Fig. 6(b)]. Contrary to the expectation that the strong-coupling limit will suppress gradually the intrapair correlation length in the first band, we observe a nonmonotonic dependence and a significant amplification of $\xi_{\text {pairl }}$ in the BEC regime. From the physical point of view, such results point out the formation of giant Cooper pairs in the first band with bosonic pairs in the second band. This coexistence of BCS and BEC condensates stems from the weak interband coupling, where the cold band serves as an almost independent reservoir of Cooper pairs. Thus, a two-band superfluid system is described as the continuous transformations from two different BCS condensates to a system where giant Cooper pairs and bosonic condensate coexist and then finally to a mixture of two BEC condensates [Fig. 7(a)]. The crossover in the cold band above discussed can be also interpreted as a density-induced BCSBEC crossover [49], when the density $n_{1}$ is tuned by the coupling in the hot band.

For larger values of $\tilde{U}_{12}>0.063$ and for $\left(k_{\mathrm{F} 1} a_{11}\right)^{-1}=$ -2 , we observe gradually a disappearance of this peak and the transition to the conventional single-band behavior of the intrapair correlation length for the first band [Figs. 6(c) and 6(d)]. The behavior of $\xi_{\text {pair2 } 2}$ at the qualitative level maintains the same dependence as for the single-band counterpart. For very strong interband interaction, the dependencies of the intrapair correlation lengths in each band on $\left(k_{\mathrm{F} 2} a_{22}\right)^{-1}$ are the same.

Increasing the intraband coupling in the first band for a given strength of the interband interaction leads to a reduction of the peak. Nevertheless, for very weak interband coupling, the effect of the amplification of the intrapair correlation length can be preserved even for $\left(k_{\mathrm{F} 1} a_{11}\right)^{-1}=-0.5$ [Fig. 7(a)].

Using the criterion of strongly overlapping Cooper pairs for the single-band system $k_{F} \xi_{\text {pair }}>2 \pi$ we can extract interesting feature of the BCS-BEC crossover in a weakly interacting two-band Fermi gas. In particular, when the value of $\left(k_{\mathrm{F} 1} a_{11}\right)^{-1}<0$ we have a rich picture of the BCS-BEC crossover evolution [Fig. 7(a)]. Initially for $\left(k_{\mathrm{F} 2} a_{22}\right)^{-1} \ll-1$ there is a mixture of two Cooper pairs condensates. Then, with the increasing of the intraband coupling toward strong coupling, the formation of giant Cooper pairs in the first band occurs. Such pairs coexist with the BEC condensate from the second band. In the extremely strong-coupling limit $\left(k_{\mathrm{F} 2} a_{22}\right)^{-1} \gg-1$, we observe the transition from the coexistence of giant Cooper pairs and BEC molecules to a 

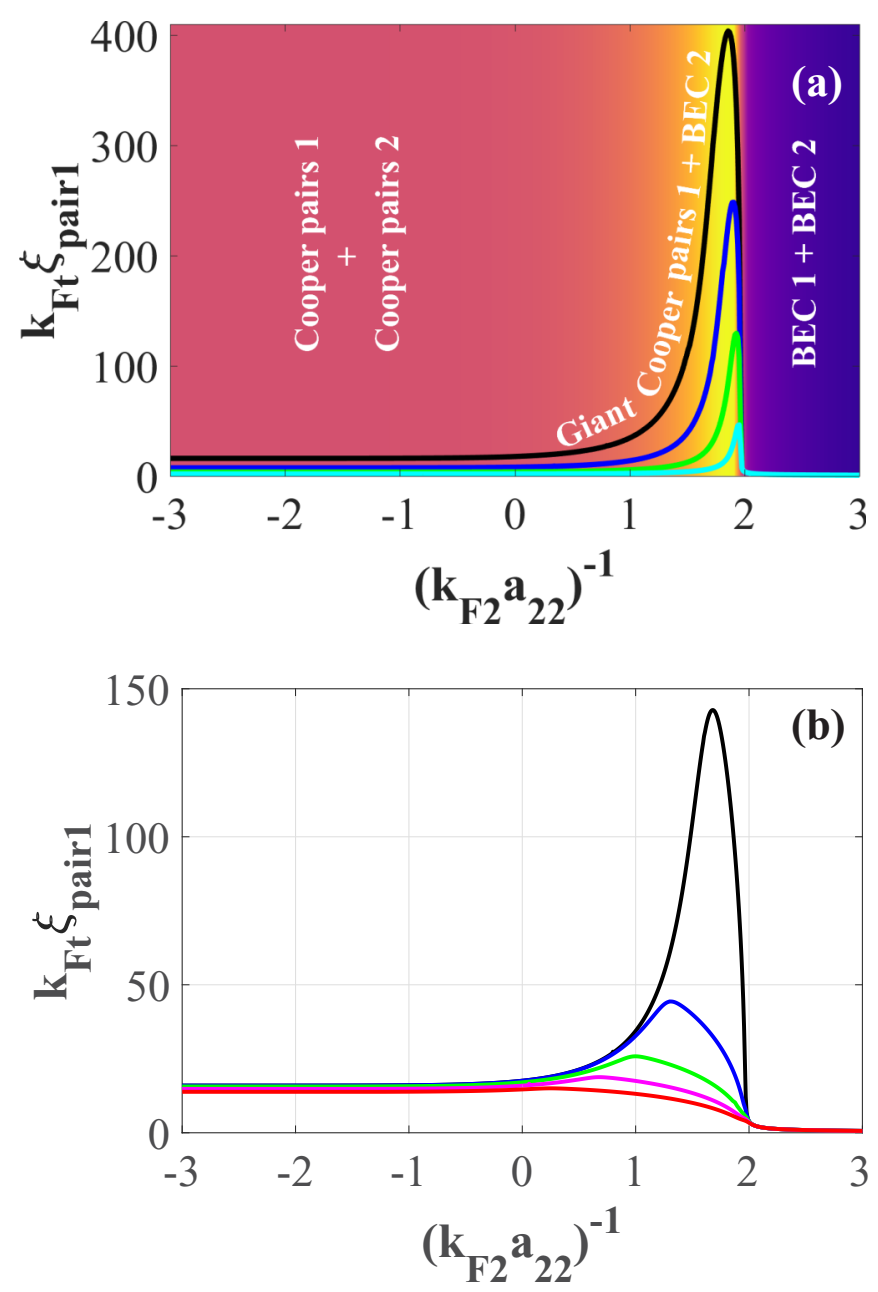

FIG. 7. (a) Evolution of the intrapair correlation length in the first band $\xi_{\text {pair } 1}$ of a very weakly interacting superfluid two-band Fermi gas with $\tilde{U}_{12}=0.001$ as a function of $\left(k_{\mathrm{F} 2} a_{22}\right)^{-1}$ for $\left(k_{\mathrm{F} 1} a_{11}\right)^{-1}=-2$ (black line), $\left(k_{\mathrm{F} 1} a_{11}\right)^{-1}=-1.5$ (blue line), $\left(k_{\mathrm{F} 1} a_{11}\right)^{-1}=-1$ (green line), and $\left(k_{\mathrm{F} 1} a_{11}\right)^{-1}=-0.5$ (cyan line) at $T=0$. (b) Temperature effect on the amplification of the intrapair correlation length $\xi_{\text {pair } 1}$ for $\tilde{U}_{12}=0.001$ with $\left(k_{\mathrm{F} 1} a_{11}\right)^{-1}=-2$. Black line is for $T=0$ (the maximum value of the intraband length corresponds to the critical temperature of the system $T_{c} \approx 0.67 T_{\mathrm{Ft}}$ and to the critical temperature of the first band $T_{c 1} \approx 0.0007 T_{\mathrm{Ft}}$ ), blue line is for $T=0.005 E_{\mathrm{Ft}}$ (maximum of $\xi_{\text {pair1 }}$ corresponds to $T_{c} \approx 0.523 T_{\mathrm{Ft}}$ and $T_{c 1} \approx 0.0043 T_{\mathrm{Ft}}$ ), green line for $T=0.01 E_{\mathrm{Ft}}\left(T_{c} \approx 0.41 T_{\mathrm{Ft}}\right.$ and $\left.T_{c 1} \approx 0.0092 T_{\mathrm{Ft}}\right)$, cyan line for $T=0.015 E_{\mathrm{Ft}}\left(T_{c} \approx 0.3 T_{\mathrm{Ft}}\right.$ and $\left.T_{c 1} \approx 0.014 T_{\mathrm{Ft}}\right)$, and red line is for $T=0.02 E_{\mathrm{Ft}}\left(T_{c} \approx 0.188 T_{\mathrm{Ft}}\right.$ and $\left.T_{c 1} \approx 0.02 T_{\mathrm{Ft}}\right)$.

two-component Bose condensate with coinciding intrapair correlation lengths.

We move now to discuss the effects of temperature on the phenomenon of giant Cooper-pair formation. A slight increase of the temperature decreases the magnitude of the giant Cooper-pair effect dramatically, and shifts slightly the position of the peak to smaller values of $\left(k_{\mathrm{F} 2} a_{22}\right)^{-1}$ [Fig. 7(b)]. The explanation of such a behavior will be provided below.

Investigation of the temperature dependencies of the energy gaps show an anomalous behavior of $\Delta_{2}$ in the case of weak interband coupling (Fig. 8). The occurrence of the
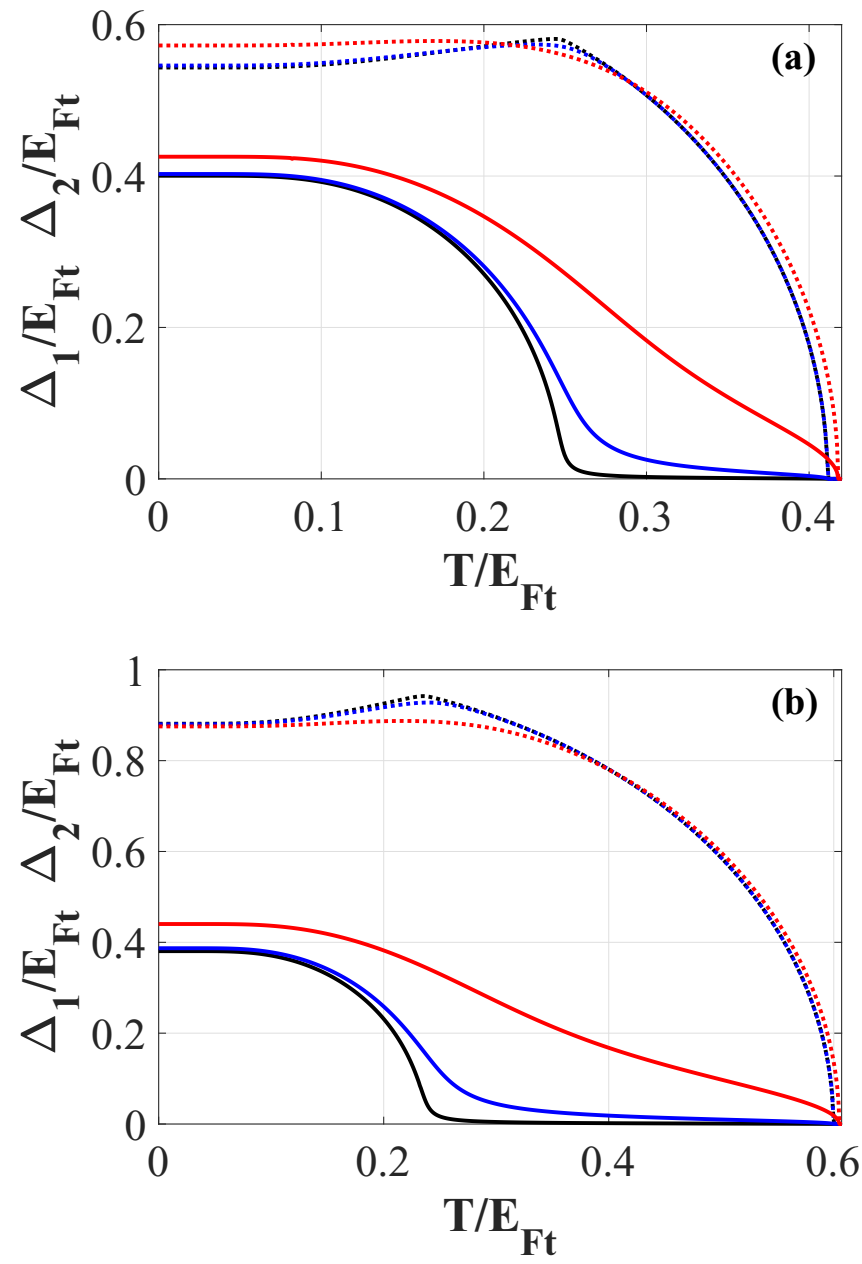

FIG. 8. Temperature dependence of the energy gaps $\Delta_{1}$ (solid line) and $\Delta_{2}$ (dotted line) of a two-band superfluid Fermi gas with $\tilde{U}_{12}=0.001$ (black line), $\tilde{U}_{12}=0.01$ (blue line), and $\tilde{U}_{12}=0.1$ (red line) for $\left(k_{\mathrm{F} 1} a_{11}\right)^{-1}=-0.25,\left(k_{\mathrm{F} 2} a_{22}\right)^{-1}=1$ (a) and $\left(k_{\mathrm{F} 1} a_{11}\right)^{-1}=0$, $\left(k_{\mathrm{F} 2} a_{22}\right)^{-1}=1.5(\mathrm{~b})$.

kink is directly connected with the increasing of the intrapair correlation length in the first band. Moreover, the temperature dependencies of the chemical potential (not shown) also have a kink for the same values of $T$. Numerical analysis shows that the effect is more pronounced when the values of energy gaps become comparable. At the same time, we recall that the strong enhancement of $\xi_{\text {pairl }}$ is realized in the BEC limit of the second band at $T=0$ and for vanishing $U_{12}$ [Fig. 7(a)]. The increasing of the temperature, of the strong interband interaction, or a strong intraband coupling in the first band lead to the suppression of the intrapair correlation length [Figs. 6 and 7(b)]. Thus, in order to have simultaneously comparable energy gaps in the two bands and the intrapair correlation length amplification effect, the second band should be in the strong-coupling regime [for values of $\left(k_{\mathrm{F} 2} a_{22}\right)^{-1}$, where we have the strong enhancement of $\left.\xi_{\text {pairl }}\right]$ and the first band near the unitarity point $\left(k_{\mathrm{F} 1} a_{11}\right)^{-1} \approx 0$ (this yields $\left.\Delta_{1} \cong \Delta_{2}\right)$. Other conditions eliminate the nonmonotonic dependence of $\Delta_{2}(T)$, leading to the conventional BCS-type behavior of the energy gap in the second band, or decrease too much the magnitude of the peak. In turn, with the increase of the 
temperature, the suppression of the peak in $\xi_{\text {pair1 }}$ becomes important when the temperature is in the vicinity of the critical temperature of the first cold band $T_{c 1}$, which is very small for the parameters here considered [see the legend in the Fig. 7(b)].

Based on this, we can claim that the decreasing rate of the intrapair correlation length in the first band with temperature is determined by the width of the temperature interval, where the non-BCS behavior of the second gap is realized (or, equivalently, where the first energy gap is not strongly suppressed). Since Fig. 7(b) corresponds to the system with $\left(k_{\mathrm{F} 1} a_{11}\right)^{-1}=$ -2 , i.e., the BCS regime for the first band, we have the small temperature interval of non-BCS dependence of $\Delta_{2}$ and, as a consequence, the rapid temperature suppression of $\xi_{\text {pair } 1}$.

It is important to note that a similar behavior of the intrapair correlation lengths as a function of temperature was revealed in a two-band superconductor with very weak interband interaction [50-53]. In the absence of coupling between two superconducting condensates below the critical temperature, a hidden critical point appears at the critical temperature of the weaker band that corresponds to the divergence of the intrapair correlation length. In the case of weak interband interaction, the intrapair correlation length of the weaker band exhibits a deviation from the conventional monotonic increase with temperature, and leads to a pronounced peak close to the hidden critical point. In our calculations, the interband coupling also governs the effect but, as opposite to a two-band superconductor, a strong enhancement of the intrapair correlation length in one of the bands occurs in the strong-coupling limit, where the formation of giant Cooper pairs is not expected. Moreover, as it was shown above, this phenomenon for very weak interband interaction can be observed even at finite temperatures. We emphasize that the hidden critical-like behavior is observed in our case for strong intraband interactions, whereas in Refs. [50-53] it was reported in the weak-coupling limit.

We suggest that the experimental detection of giant Cooper pairs in the strong-coupling regime and the verification of our prediction can be done through direct imaging of vortex cores in two-component fermionic condensates or in iron-based superconductors with electroniclike concentric Fermi surfaces. Another possibility to verify our predictions would be a precise measurement of the temperature dependence of the energy gaps in two-band superfluid systems.

\section{CONCLUSIONS}

We have investigated the characteristics and have found unique properties of the BCS-BEC crossover in a two-band superfluid Fermi system in the presence of an energy shift between the bands, for different pairing strengths in the two bands. We have demonstrated the richness of the BCS-BEC crossover in such a two-band system as compared with its single-band counterpart. We have found that for vanishing interband interaction at low temperatures and in the strongcoupling regime of the second band, a two-band superfluid Fermi gas evolves to a single-band system with the full suppression of the energy gap in the first band, together with the full redistribution of particles. As a result, a giant enhancement of the intrapair correlation length of Cooper pairs in the first band occurs. In the case of finite coupling between the two condensates of the two bands, we have shown a nonmonotonic behavior of the first energy gap with a hump, the position of which is determined by the strength of the interband interaction in the second band. For weak interband coupling we have found a significant amplification of the intrapair correlation length of the first band in the BEC regime for the second band at zero and finite temperatures, which indicates the coexistence of giant Cooper pairs and bosonic condensate in a two-band superfluid system. We have revealed that such an effect can produce an unusual nonmonotonic temperature dependence of the second energy gap with the presence of a maximum for nonzero temperatures. Our predictions can be verified in two-component atomic condensates and in some iron-based superconductors having electronlike or holelike concentric bands with low filling and weak interband interaction, via STM investigations of vortex cores or by studying the temperature behavior of the energy gaps.

\section{ACKNOWLEDGMENTS}

This work was supported by the Italian MIUR through the PRIN 2015 program (Contract No. 2015C5SEJJ001). H.T. was supported by Grant-in-Aid for JSPS fellows (Grant No. 17J03975). We thank A. Vagov, M. V. Milošević, and S. Klimin for discussions.

\section{APPENDIX A: EQUATIONS FOR THE CRITICAL TEMPERATURE, CHEMICAL POTENTIAL, AND PARTICLE DENSITIES}

\section{Equations in dimensionless form}

For the numerical solutions of Eqs. (3)-(6) of the main text, it is convenient to measure momenta and energies in units of the the total Fermi momentum $k_{\mathrm{Ft}}$ and total Fermi energy $E_{\mathrm{Ft}}$, respectively. In this way, the matrix gap equation (3) in dimensionless form reads as

$$
\begin{gathered}
\tilde{\Delta}_{1} \int_{0}^{k_{0} / k_{\mathrm{Ft}}} x^{2} \frac{\tanh \frac{\sqrt{\left(x^{2}-\tilde{\mu}\right)^{2}+\tilde{\Delta}_{1}^{2}}}{2 t}}{\sqrt{\left(x^{2}-\tilde{\mu}\right)^{2}+\tilde{\Delta}_{1}^{2}}} d x \\
=\frac{A_{22}}{W} \tilde{\Delta}_{1}-\frac{3}{4} \frac{\tilde{U}_{12}}{W}\left(\frac{k_{\mathrm{Ft}}}{k_{0}}\right)^{2} \tilde{\Delta}_{2},
\end{gathered}
$$

$$
\begin{aligned}
& \tilde{\Delta}_{2} \int_{0}^{k_{0} / k_{\mathrm{Ft}}} x^{2} \frac{\tanh \frac{\sqrt{\left(x^{2}-\tilde{\mu}+\frac{E_{\mathrm{g}}}{E_{\mathrm{Ft}}}\right)^{2}+\tilde{\Delta}_{2}^{2}}}{2 t}}{\sqrt{\left(x^{2}-\tilde{\mu}+\frac{E_{\mathrm{g}}}{E_{\mathrm{Ft}}}\right)^{2}+\tilde{\Delta}_{2}^{2}}} d x \\
& =\frac{A_{11}}{W} \tilde{\Delta}_{2}-\frac{3}{4} \frac{\tilde{U}_{21}}{W}\left(\frac{k_{\mathrm{Ft}}}{k_{0}}\right)^{2} \tilde{\Delta}_{1},
\end{aligned}
$$

where $x=k / k_{\mathrm{Ft}}, \tilde{\Delta}_{i}=\Delta_{i} / E_{\mathrm{Ft}}, \tilde{\mu}=\mu / E_{\mathrm{Ft}}, t=T / E_{\mathrm{Ft}}$, and we have introduced the notations $A_{i i}=\left(\frac{k_{0}}{k_{\mathrm{Ft}}}-\frac{\pi}{2 k_{F i} a_{i i}} \frac{k_{F i}}{k_{\mathrm{Ft}}}\right)^{-1}$ and $W=A_{11} A_{22}-\frac{9}{16} \tilde{U}_{12} \tilde{U}_{21}\left(\frac{k_{\mathrm{Ft}}}{k_{0}}\right)^{4}$. 
Note that in Eqs. (A1) and (A2) we have used Eq. (9) to express $U_{i i}$ in terms of the scattering length $a_{i i}$. Similarly, the particle-number equation (5) with $n_{i}$ given by Eq. (6) can be cast in the dimensionless form

$$
\begin{aligned}
\frac{2}{3}= & \int_{0}^{k_{0} / k_{\mathrm{Ft}}} x^{2}\left(1-\frac{x^{2}-\tilde{\mu}}{\sqrt{\left(x^{2}-\tilde{\mu}\right)^{2}+\tilde{\Delta}_{1}^{2}}} \tanh \frac{\sqrt{\left(x^{2}-\tilde{\mu}\right)^{2}+\tilde{\Delta}_{1}^{2}}}{2 t}\right) d x \\
& +\int_{0}^{k_{0} / k_{\mathrm{Ft}}} x^{2}\left(1-\frac{x^{2}-\tilde{\mu}+\frac{E_{\mathrm{g}}}{E_{\mathrm{Ft}}}}{\sqrt{\left(x^{2}-\tilde{\mu}+\frac{E_{\mathrm{g}}}{E_{\mathrm{Ft}}}\right)^{2}+\tilde{\Delta}_{2}^{2}}} \tanh \frac{\sqrt{\left(x^{2}-\tilde{\mu}+\frac{E_{\mathrm{g}}}{E_{\mathrm{Ft}}}\right)^{2}+\tilde{\Delta}_{2}^{2}}}{2 t}\right) d x,
\end{aligned}
$$

where we have neglected the free-particle contribution for $k>k_{0}$ since it is exponentially small in the limit $\frac{k_{0}}{k_{\mathrm{Ft}}} \gg 1$ of relevance to our work.

\section{Mean field critical temperature and chemical potential at $T_{\mathrm{c}}$}

In the vicinity of the critical temperature we can linearize the system of Eqs. (A1)-(A3) and from the condition of solvability we obtain the equation for the critical temperature and the particle densities (from now on, we will omit the tilde symbol for the dimensionless energy gaps and chemical potential)

$$
\begin{gathered}
\left(\int_{0}^{k_{0} / k_{\mathrm{Ft}}} \frac{x^{2} \tanh \frac{x^{2}-\mu}{2 t}}{x^{2}-\mu} d x-\frac{A_{22}}{W}\right)\left(\int_{0}^{k_{0} / k_{\mathrm{Ft}}} \frac{x^{2} \tanh \frac{x^{2}-\mu+\frac{E_{\mathrm{g}}}{E_{\mathrm{Ft}}}}{2}}{x^{2}-\mu+\frac{E_{\mathrm{g}}}{E_{\mathrm{Ft}}}} d x-\frac{A_{11}}{W}\right)-\frac{9}{16} \frac{\tilde{U}_{12} \tilde{U}_{21}}{W^{2}}\left(\frac{k_{\mathrm{Ft}}}{k_{0}}\right)^{4}=0, \\
\frac{2}{3}=\int_{0}^{k_{0} / k_{\mathrm{Ft}}} x^{2}\left(1-\tanh \frac{x^{2}-\mu}{2 t}\right) d x+\int_{0}^{k_{0} / k_{\mathrm{Ft}}} x^{2}\left(1-\tanh \frac{x^{2}-\mu+\frac{E_{\mathrm{g}}}{E_{\mathrm{Ft}}}}{2 t}\right) d x .
\end{gathered}
$$

Based on asymptotic expansions for $\frac{k_{0}}{k_{\mathrm{Ft}}} \gg 1$,

$$
\frac{k_{0}}{k_{\mathrm{Ft}}}-\frac{A_{11}}{W} \approx \frac{\pi}{2 k_{\mathrm{F} 1} a_{11}} \frac{k_{\mathrm{F} 1}}{k_{\mathrm{Ft}}}
$$

and

$$
\frac{k_{0}}{k_{\mathrm{Ft}}}-\frac{A_{22}}{W} \approx \frac{\pi}{2 k_{\mathrm{F} 2} a_{22}} \frac{k_{\mathrm{F} 2}}{k_{\mathrm{Ft}}}
$$

we rewrite Eqs. (A4) and (A5) extending the limit of the integration up to infinity and thereby eliminating the cutoff momentum dependence for the determination of the critical temperature

$$
\begin{gathered}
{\left[\int_{0}^{+\infty}\left(1-\frac{\left.x^{2} \tanh \frac{x^{2}-\mu}{2 t}\right)}{x^{2}-\mu}\right) d x-\frac{\pi}{2 k_{\mathrm{F} 1} a_{11}} \frac{k_{\mathrm{F} 1}}{k_{\mathrm{Ft}}}\right]\left[\int_{0}^{+\infty}\left(1-\frac{x^{2} \tanh \frac{x^{2}-\mu+\frac{E_{\mathrm{g}}}{E_{\mathrm{Ft}}}}{2 t}}{x^{2}-\mu+\frac{E_{\mathrm{g}}}{E_{\mathrm{Ft}}}}\right) d x-\frac{\pi}{2 k_{\mathrm{F} 2} a_{22}} \frac{k_{\mathrm{F} 2}}{k_{\mathrm{Ft}}}\right]-\frac{9}{16} \tilde{U}_{12} \tilde{U}_{21}=0,} \\
-t^{\frac{3}{2}} \Gamma\left(\frac{3}{2}\right) \operatorname{Li}_{\frac{3}{2}}\left(-e^{\frac{\mu}{t}}\right)-t^{\frac{3}{2}} \Gamma\left(\frac{3}{2}\right) \operatorname{Li}_{\frac{3}{2}}\left(-e^{\frac{\mu-\frac{E_{\mathrm{Ft}}}{t}}{t}}\right)=\frac{2}{3},
\end{gathered}
$$

where the integration of Eq. (A5) was performed in terms of the polylogarithm function $\mathrm{Li}_{s}(z)$ and the gamma function $\Gamma(z)$. Equation (A9) can be simplified in the BCS limit, for which $t \ll 1$, by using

$$
\lim _{t \rightarrow 0}\left[-t^{\frac{3}{2}} \operatorname{Li}_{\frac{3}{2}}\left(-e^{\frac{\mu}{t}}\right)\right]=\frac{4}{3 \sqrt{\pi}} \mu^{\frac{3}{2}}
$$

so that Eq. (A9) simplifies to

$$
\mu^{\frac{3}{2}}+\left(\mu-\frac{E_{\mathrm{g}}}{E_{\mathrm{Ft}}}\right)^{\frac{3}{2}}=1
$$

which has the approximated solution

$$
\mu \approx \frac{1}{3} \frac{3 \sqrt{1-\frac{E_{\mathrm{g}}}{E_{\mathrm{Ft}}}}-2\left(1-\frac{E_{\mathrm{g}}}{E_{\mathrm{Ft}}}\right)^{\frac{3}{2}}+3}{1+\sqrt{1-\frac{E_{\mathrm{g}}}{E_{\mathrm{Ft}}}}},
$$

which, as expected, just coincides with $E_{\mathrm{F} 1}$. For a zero-energy shift $E_{\mathrm{g}}=0$ and in the weak-coupling limit, Eq. (A8) reduces to

$$
\begin{aligned}
& \left(\ln \frac{2^{\frac{2}{3}} \pi e^{2} t}{8 e^{\gamma}}-\frac{\pi}{2 k_{\mathrm{F} 1} a_{11}} \frac{k_{\mathrm{F} 1}}{k_{\mathrm{Ft}}}\right)\left(\ln \frac{2^{\frac{2}{3}} \pi e^{2} t}{8 e^{\gamma}}-\frac{\pi}{2 k_{\mathrm{F} 2} a_{22}} \frac{k_{\mathrm{F} 2}}{k_{\mathrm{Ft}}}\right) \\
& -\frac{9}{16} \tilde{U}_{12} \tilde{U}_{21}=0,
\end{aligned}
$$



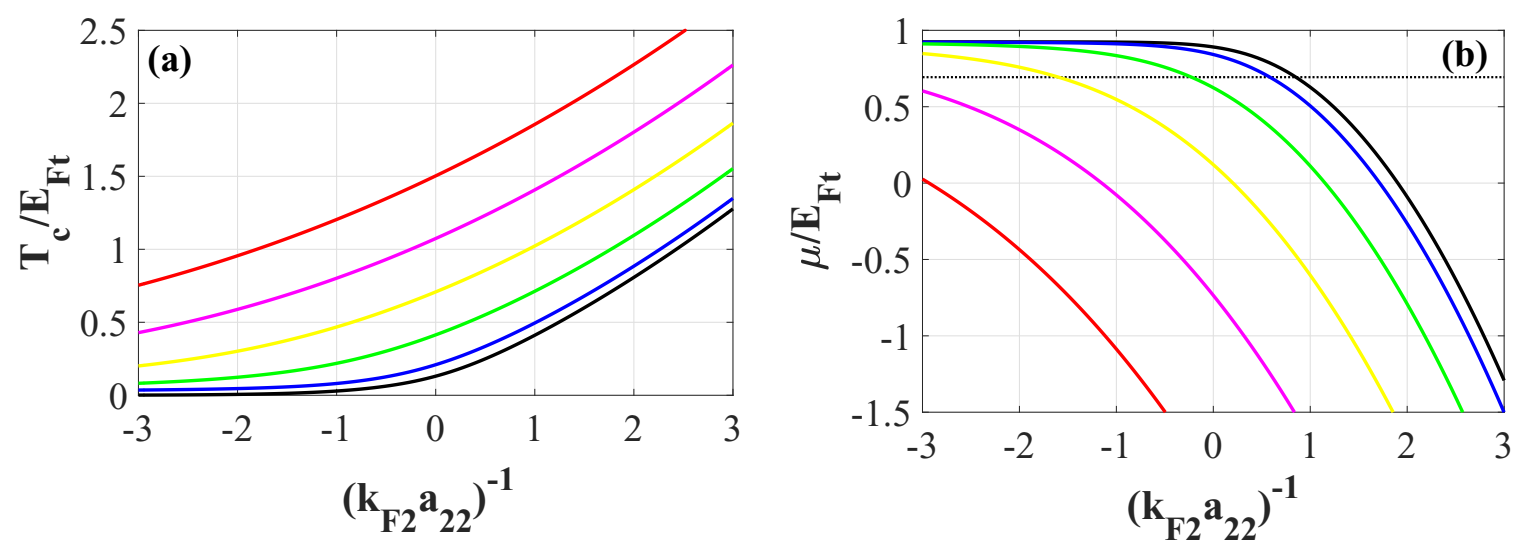

FIG. 9. (a) The critical temperature $T_{\mathrm{c}}$ and (b) the chemical potential $\mu$ (at $T=T_{\mathrm{c}}$ ) vs $\left(k_{\mathrm{F} 2} a_{22}\right)^{-1}$ for different interband couplings $\tilde{U}_{12}=0$ (black line), $\tilde{U}_{12}=1$ (blue line), $\tilde{U}_{12}=2$ (green line), $\tilde{U}_{12}=3$ (yellow line), $\tilde{U}_{12}=4$ (magenta line), $\tilde{U}_{12}=5$ (red line) with fixed value of the scattering length in the first band $\left(k_{\mathrm{F} 1} a_{11}\right)^{-1}=-2$. The dotted black line is the energy shift $E_{\mathrm{g}}$ between bands in units of $E_{\mathrm{Ft}}$.

where $\gamma=0.577 \ldots$ is the Euler-Mascheroni constant and where the simplified equation for the weak-coupling limit (A11) gives the exact value of the chemical potential $\mu=2^{-\frac{2}{3}}$. Formally, Eq. (A13) has the same form as the equation for the determination of the critical temperature of a clean two-band superconductor $[1,2,54]$ with the corresponding solution

$$
\begin{aligned}
\frac{T_{c}}{E_{\mathrm{Ft}}}= & \frac{8 e^{\gamma}}{2^{\frac{2}{3}} \pi e^{2}} \exp \left[\frac { 1 } { 4 } \left(\frac{\pi}{k_{\mathrm{F} 1} a_{11}} \frac{k_{\mathrm{F} 1}}{k_{\mathrm{Ft}}}+\frac{\pi}{k_{\mathrm{F} 2} a_{22}} \frac{k_{\mathrm{F} 2}}{k_{\mathrm{Ft}}}\right.\right. \\
& +\sqrt{\left.\left.\left(\frac{\pi}{k_{\mathrm{F} 1} a_{11}} \frac{k_{\mathrm{F} 1}}{k_{\mathrm{Ft}}}-\frac{\pi}{k_{\mathrm{F} 2} a_{22}} \frac{k_{\mathrm{F} 2}}{k_{\mathrm{Ft}}}\right)^{2}+9 \tilde{U}_{12} \tilde{U}_{21}\right)\right] .}
\end{aligned}
$$

Still in the BCS limit, but considering now the limit where $E_{\mathrm{g}}$ approaches $E_{\mathrm{F} 1}$ from below, the hyperbolic tangent function in the second brackets of Eq. (A8) can be set to one. This yields the equation

$$
\begin{aligned}
& \left(\ln \frac{\pi e^{2} t}{8 \mu_{s} e^{\gamma}}-\frac{\pi}{2 k_{\mathrm{F} 1} a_{11}} \frac{k_{\mathrm{F} 1}}{k_{\mathrm{Ft}}}\right)\left(\frac{\pi}{2} \sqrt{\frac{E_{\mathrm{g}}}{E_{\mathrm{Ft}}}-1}-\frac{\pi}{2 k_{\mathrm{F} 2} a_{22}} \frac{k_{\mathrm{F} 2}}{k_{\mathrm{Ft}}}\right) \\
& -\frac{9}{16} \tilde{U}_{12} \tilde{U}_{21}=0
\end{aligned}
$$

with solution

$$
\frac{T_{c}}{E_{\mathrm{Ft}}}=\frac{8 \mu e^{\gamma}}{\pi e^{2}} \exp \left(\frac{\pi}{2 k_{\mathrm{F} 1} a_{11}} \frac{k_{\mathrm{F} 1}}{k_{\mathrm{Ft}}}+\frac{9}{16} \frac{\tilde{U}_{12} \tilde{U}_{21}}{\frac{\pi}{2} \sqrt{\frac{E_{\mathrm{g}}}{E_{\mathrm{Ft}}}-1}-\frac{\pi}{2 k_{\mathrm{F} 2} a_{22}} \frac{k_{\mathrm{F} 2}}{k_{\mathrm{Ft}}}}\right) .
$$

We can see that in this case, and for vanishing interaction between bands, the critical temperature of the system does not depend on $\left(k_{\mathrm{F} 2} a_{22}\right)^{-1}$, and in the BCS regime is determined by the first band only.

Now, we analyze numerically the general behavior of the critical temperature and of the corresponding value of the chemical potential for a two-band superfluid Fermi gas. As we can see with the increasing of the interband coupling strength, we observe an increase of the critical temperature with a corresponding decrease of the chemical potential (Fig. 9). Figure 10 shows the distribution of particle densities between bands with the increasing of the interband coupling strength near the critical temperature. For strong interband interaction, $n_{1}$ and $n_{2}$ exhibit a tendency to equalize the particle densities in each band toward the BEC limit of the second band. Comparing with Fig. 5 in Ref. [40] one can see that pairing fluctuations associated with both interband and intraband couplings reduced significantly the effects of the particle interband distribution in the vicinity of $T_{c}$.

Also, it should be noted that the effect of the interband interaction is the most distinct for low temperatures, where even small changes of $U_{12}$ give rise to a perceptible effect for the energy gaps and the chemical potential (see Fig. 2 in Sec. III.).

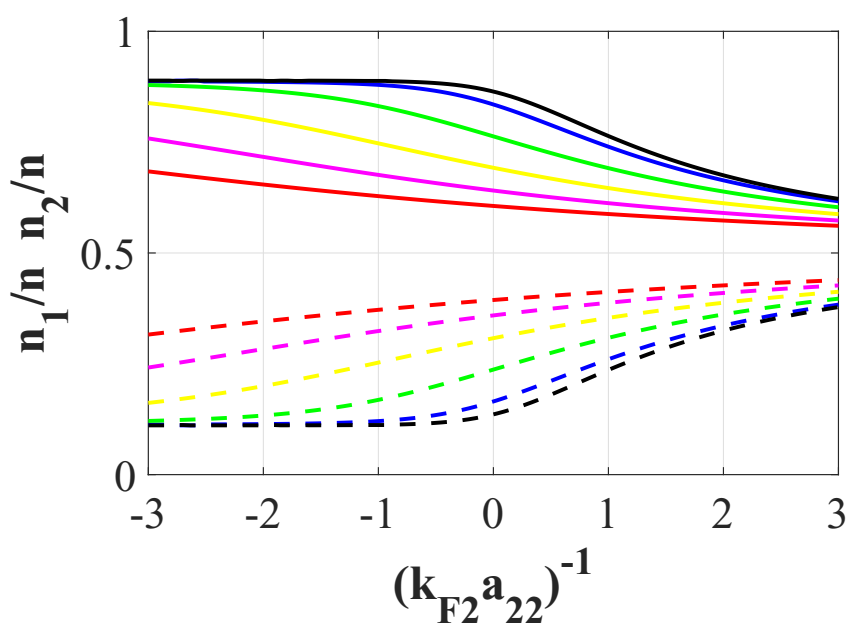

FIG. 10. Particle densities in the first (solid lines) and in the second (dashed lines) bands for different interband couplings $\tilde{U}_{12}=$ 0 (black line), $\tilde{U}_{12}=1$ (blue line), $\tilde{U}_{12}=2$ (green line), $\tilde{U}_{12}=$ 3 (yellow line), $\tilde{U}_{12}=4$ (magenta line), $\tilde{U}_{12}=5$ (red line) with $\left(k_{\mathrm{F} 1} a_{11}\right)^{-1}=-2$. 


\section{APPENDIX B: COMPARISON OF APPROACHES: FIXED INTRABAND COUPLINGS RATIO VS FIXED INTRABAND COUPLING IN ONE OF THE BANDS}

In this Appendix we compare two different approaches for the description of the BCS-BEC crossover characteristics based on a mean field theory and their effect on the results. We start from the simple case: two-band superfluid Fermi gas with vanishing interaction between bands at zero temperature. At the beginning we consider the approach with a fixed ratio of intraband couplings $\rho=\tilde{U}_{22} / \tilde{U}_{11}$. This definition gives the relation between scattering lengths in each band

$$
\frac{\pi}{2 k_{\mathrm{F} 1} a_{11}} \frac{k_{\mathrm{F} 1}}{k_{\mathrm{Ft}}}=-\frac{k_{0}}{k_{\mathrm{Ft}}}(\rho-1)+\frac{\pi}{2 k_{\mathrm{F} 2} a_{22}} \frac{k_{\mathrm{F} 2}}{k_{\mathrm{Ft}}} \rho .
$$

Substituting Eq. (B1) into Eqs. (3)-(5) for the energy gaps and the particle densities and taking into account dimensionless notations we have again

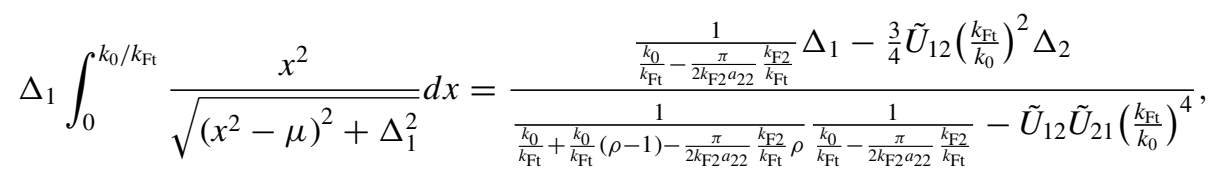

$$
\begin{aligned}
& \Delta_{2} \int_{0}^{k_{0} / k_{\mathrm{Ft}}} \frac{x^{2}}{\sqrt{\left(x^{2}-\mu+\frac{E_{\mathrm{g}}}{E_{\mathrm{Ft}}}\right)^{2}+\Delta_{2}^{2}}} d x=\frac{\frac{1}{\frac{k_{0}}{k_{\mathrm{Ft}}}+\frac{k_{0}}{k_{\mathrm{Ft}}}(\rho-1)-\frac{\pi}{2 k_{\mathrm{F} 2} a_{22}} \frac{k_{\mathrm{F} 2}}{k_{\mathrm{Ft}}} \rho} \Delta_{2}-\frac{3}{4} \tilde{U}_{21}\left(\frac{k_{\mathrm{Ft}}}{k_{0}}\right)^{2} \Delta_{1}}{\frac{1}{\frac{k_{0}}{k_{\mathrm{Ft}}}+\frac{k_{0}}{k_{\mathrm{Ft}}}(\rho-1)-\frac{\pi}{2 k_{\mathrm{F} 2} a_{22}} \frac{k_{\mathrm{F} 2}}{k_{\mathrm{Ft}}} \rho} \frac{k_{0}}{k_{\mathrm{Ft}}-\frac{\pi}{2 k_{\mathrm{F} 2} a_{22}}}-\frac{k_{\mathrm{F} 2}}{k_{\mathrm{Ft}}}}-\frac{9}{16} \tilde{U}_{12} \tilde{U}_{21}\left(\frac{k_{\mathrm{Ft}}}{k_{0}}\right)^{4}, \\
& \frac{2}{3}=\int_{0}^{k_{0} / k_{\mathrm{Ft}}} x^{2}\left(1-\tanh \frac{x^{2}-\mu}{2 t}\right) d x+\int_{0}^{k_{0} / k_{\mathrm{Ft}}} x^{2}\left(1-\tanh \frac{x^{2}-\mu+\frac{E_{\mathrm{g}}}{E_{\mathrm{Ft}}}}{2 t}\right) d x .
\end{aligned}
$$

The first two equations of the above system can be integrated analytically and after a long but straightforward calculation they can be expressed via elliptic integrals

$$
\begin{aligned}
& \int_{0}^{k_{0} / k_{\mathrm{Ft}}} \frac{x^{2}}{\sqrt{\left(x^{2}-\mu\right)^{2}+\Delta_{1}^{2}}} d x=\sqrt{i \Delta_{1}+\mu}\left[F\left(\frac{k_{0}}{k_{\mathrm{Ft}}} \sqrt{\frac{i \Delta_{1}+\mu}{\Delta_{1}^{2}+\mu^{2}}}, \sqrt{\frac{i \mu+\Delta_{1}}{i \mu-\Delta_{1}}}\right)-E\left(\frac{k_{0}}{k_{\mathrm{Ft}}} \sqrt{\frac{i \Delta_{1}+\mu}{\Delta_{1}^{2}+\mu^{2}}}, \sqrt{\frac{i \mu+\Delta_{1}}{i \mu-\Delta_{1}}}\right)\right], \\
& \int_{0}^{k_{0} / k_{\mathrm{Ft}}} \frac{x^{2}}{\sqrt{\left(x^{2}-\mu+E_{\mathrm{g}}\right)^{2}+\Delta_{2}^{2}}} d x=\sqrt{i \Delta_{2}+\mu-E_{\mathrm{g}}}\left[F\left(\frac{k_{0}}{k_{\mathrm{Ft}}} \sqrt{\frac{i \Delta_{2}+\mu-E_{\mathrm{g}}}{\Delta_{2}^{2}+\left(\mu-E_{\mathrm{g}}\right)^{2}}}, \sqrt{\frac{i\left(\mu-E_{\mathrm{g}}\right)+\Delta_{2}}{i\left(\mu-E_{\mathrm{g}}\right)-\Delta_{2}}}\right)\right. \\
& \left.-E\left(\frac{k_{0}}{k_{\mathrm{Ft}}} \sqrt{\frac{i \Delta_{2}+\mu-E_{\mathrm{g}}}{\Delta_{2}^{2}+\left(\mu-E_{\mathrm{g}}\right)^{2}}}, \sqrt{\frac{i\left(\mu-E_{\mathrm{g}}\right)+\Delta_{2}}{i\left(\mu-E_{\mathrm{g}}\right)-\Delta_{2}}}\right)\right],
\end{aligned}
$$

where $F(z, v)$ and $E(z, v)$ are incomplete elliptic integrals of the first and the second kind and $i$ is the imaginary unit. This yields the system of equations for the energy gaps

$$
\begin{aligned}
& \Delta_{1} \sqrt{i \Delta_{1}+\mu}\left[F\left(\frac{k_{0}}{k_{\mathrm{Ft}}} \sqrt{\frac{i \Delta_{1}+\mu}{\tilde{\Delta}_{1}^{2}+\mu^{2}}}, \sqrt{\frac{i \mu+\Delta_{1}}{i \mu-\Delta_{1}}}\right)-E\left(\frac{k_{0}}{k_{\mathrm{Ft}}} \sqrt{\frac{i \Delta_{1}+\mu}{\Delta_{1}^{2}+\mu^{2}}}, \sqrt{\frac{i \mu+\tilde{\Delta}_{1}}{i \mu-\Delta_{1}}}\right)\right] \\
& =\frac{\frac{1}{\frac{k_{0}}{k_{\mathrm{Ft}}}-\frac{\pi}{2 k_{\mathrm{F} 2} a_{22}} \frac{k_{\mathrm{F} 2}}{k_{\mathrm{Ft}}}} \Delta_{1}-\frac{3}{4} \tilde{U}_{12}\left(\frac{k_{\mathrm{Ft}}}{k_{0}}\right)^{2} \Delta_{2}}{\frac{1}{\frac{k_{0}}{k_{\mathrm{Ft}}}+\frac{k_{0}}{k_{\mathrm{Ft}}}(\rho-1)-\frac{\pi}{2 k_{\mathrm{F} 2} a_{22}} \frac{k_{\mathrm{F} 2}}{k_{\mathrm{Ft}}} \rho} \frac{1}{\frac{k_{0}}{k_{\mathrm{Ft}}}-\frac{\pi}{2 k_{\mathrm{F} 2} a_{22}} \frac{k_{\mathrm{F} 2}}{k_{\mathrm{Ft}}}}-\frac{9}{16} \tilde{U}_{12} \tilde{U}_{21}\left(\frac{k_{\mathrm{Ft}}}{k_{0}}\right)^{4}}, \\
& \Delta_{2} \sqrt{i \Delta_{2}+\mu-E_{\mathrm{g}}}\left[F\left(\frac{k_{0}}{k_{\mathrm{Ft}}} \sqrt{\frac{i \Delta_{2}+\mu-E_{\mathrm{g}}}{\Delta_{2}^{2}+\left(\mu-E_{\mathrm{g}}\right)^{2}}}, \sqrt{\frac{i\left(\mu-E_{\mathrm{g}}\right)+\Delta_{2}}{i\left(\mu-E_{\mathrm{g}}\right)-\Delta_{2}}}\right)-E\left(\frac{k_{0}}{k_{\mathrm{Ft}}} \sqrt{\frac{i \Delta_{2}+\mu-E_{\mathrm{g}}}{\tilde{\Delta}_{2}^{2}+\left(\mu-E_{\mathrm{g}}\right)^{2}}}, \sqrt{\frac{i\left(\mu-E_{\mathrm{g}}\right)+\Delta_{2}}{i\left(\mu-E_{\mathrm{g}}\right)-\Delta_{2}}}\right)\right] \\
& =\frac{\frac{1}{\frac{k_{0}}{k_{\mathrm{Ft}}}+\frac{k_{0}}{k_{\mathrm{Ft}}}(\rho-1)-\frac{\pi}{2 k_{\mathrm{F} 2} a_{22}} \frac{k_{\mathrm{F} 2}}{k_{\mathrm{Ft}}} \rho} \Delta_{2}-\frac{3}{4} \tilde{U}_{21}\left(\frac{k_{\mathrm{Ft}}}{k_{0}}\right)^{2} \Delta_{1}}{\frac{1}{\frac{k_{0}}{k_{\mathrm{Ft}}}+\frac{k_{0}}{k_{\mathrm{Ft}}}(\rho-1)-\frac{\pi}{2 k_{\mathrm{F} 2} a_{22}} \frac{k_{\mathrm{F} 2}}{k_{\mathrm{Ft}}} \rho} \frac{1}{\frac{k_{0}}{k_{\mathrm{Ft}}}-\frac{\pi}{2 k_{\mathrm{F} 2} a_{22} 2} \frac{k_{\mathrm{F} 2}}{k_{\mathrm{Ft}}}}-\frac{9}{16} \tilde{U}_{12} \tilde{U}_{21}\left(\frac{k_{\mathrm{Ft}}}{k_{0}}\right)^{4}} .
\end{aligned}
$$


For large values of $\frac{k_{0}}{k_{\mathrm{Et}}} \gg 1$, we power expand the incomplete elliptic integrals of the first and the second kind via full elliptic integrals of the first and second kinds $F(v)$ and $E(v)$ :

$$
\begin{gathered}
F\left(\frac{k_{0}}{k_{\mathrm{Ft}}} \sqrt{\frac{i \Delta_{1}+\mu}{\tilde{\Delta}_{1}^{2}+\mu^{2}}}, \sqrt{\frac{i \mu+\Delta_{1}}{i \mu-\Delta_{1}}}\right)-E\left(\frac{k_{0}}{k_{\mathrm{Ft}}} \sqrt{\frac{i \Delta_{1}+\mu}{\Delta_{1}^{2}+\mu^{2}}}, \sqrt{\frac{i \mu+\Delta_{1}}{i \mu-\Delta_{1}}}\right) \approx \frac{1}{\sqrt{i \Delta_{1}+\mu}} \frac{k_{0}}{k_{\mathrm{Ft}}}+i K\left(i \sqrt{\frac{2 \Delta_{1}}{i \mu-\Delta_{1}}}\right) \\
-\sqrt{\frac{i \mu-\Delta_{1}}{i \mu+\Delta_{1}}}\left[\frac{i \mu+\Delta_{1}}{i \mu-\tilde{\Delta}_{1}} E\left(\sqrt{\frac{i \mu-\Delta_{1}}{i \mu+\Delta_{1}}}\right)-\sqrt{\frac{i \mu+\Delta_{1}}{i \mu-\Delta_{1}}} E\left(\sqrt{\frac{i \mu+\Delta_{1}}{i \mu-\Delta_{1}}}\right)-\frac{2 \Delta_{1}}{i \mu-\Delta_{1}} K\left(\sqrt{\frac{i \mu-\Delta_{1}}{i \mu+\Delta_{1}}}\right)\right] .
\end{gathered}
$$

The same asymptotic expansion can be performed for the left part of Eq. (B8). For the right-hand side of Eqs. (B7) and (B8), expansions give

$$
\begin{aligned}
& \frac{\frac{1}{\frac{k_{0}}{k_{\mathrm{Ft}}}-\frac{\pi}{2 k_{\mathrm{F} 2} a_{22}} \frac{k_{\mathrm{F}}}{k_{\mathrm{Ft}}}} \Delta_{1}-\frac{3}{4} \tilde{U}_{12}\left(\frac{k_{\mathrm{Ft}}}{k_{0}}\right)^{2} \Delta_{2}}{\frac{1}{\frac{k_{0}}{k_{\mathrm{Ft}}}+\frac{k_{0}}{k_{\mathrm{Ft}}}(\rho-1)-\frac{\pi}{2 k_{\mathrm{F} 2} a_{22}} \frac{k_{\mathrm{F} 2}}{k_{\mathrm{Ft}}} \rho} \frac{1}{\frac{k_{0}}{k_{\mathrm{Ft}}}-\frac{\pi}{2 k_{\mathrm{F} 2} a_{22}} \frac{k_{\mathrm{F} 2}}{k_{\mathrm{Ft}}}}-\frac{9}{16} \tilde{U}_{12} \tilde{U}_{21}\left(\frac{k_{\mathrm{F}}}{k_{0}}\right)^{4}} \approx \rho \frac{k_{0}}{k_{\mathrm{Ft}}} \Delta_{1}-\frac{\pi}{2 k_{\mathrm{F} 2} a_{22}} \frac{k_{\mathrm{F} 2}}{k_{\mathrm{Ft}}} \Delta_{1}-\frac{3}{4} \rho \tilde{U}_{12} \Delta_{2}, \\
& \frac{\frac{1}{\frac{k_{0}}{k_{\mathrm{Ft}}}+\frac{k_{0}}{k_{\mathrm{Ft}}}(\rho-1)-\frac{\pi}{2 \mathrm{~F}_{\mathrm{F}} a_{22} 2} \frac{k_{\mathrm{F} 2}}{k_{\mathrm{Ft}}} \rho} \Delta_{2}-\frac{3}{4} \tilde{U}_{21}\left(\frac{k_{\mathrm{Ft}}}{k_{0}}\right)^{2} \Delta_{1}}{\frac{k_{0}}{k_{\mathrm{Ft}}}+\frac{k_{0}}{k_{\mathrm{Ft}}}(\rho-1)-\frac{\pi}{2 k_{\mathrm{F} 2} a_{22} 2} \frac{k_{\mathrm{F} 2}}{k_{\mathrm{Ft}}} \frac{k_{0}}{\frac{k_{0}}{\mathrm{k}_{\mathrm{Ft}}}-\frac{\pi}{2 k_{\mathrm{F} 2} a_{22} 2} \frac{k_{\mathrm{F} 2}}{k_{\mathrm{Ft}}}}-\frac{9}{16} \tilde{U}_{12} \tilde{U}_{21}\left(\frac{k_{\mathrm{Ft}}}{k_{0}}\right)^{4}} \approx \frac{k_{0}}{k_{\mathrm{Ft}}} \Delta_{2}-\frac{\pi}{2 k_{\mathrm{F} 2} a_{22}} \frac{k_{\mathrm{F} 2}}{k_{\mathrm{Ft}}} \Delta_{2}-\frac{3}{4} \rho \tilde{U}_{21} \Delta_{1} .
\end{aligned}
$$

After substitutions we finally obtain

$$
\begin{aligned}
& \frac{i \Delta_{1} K\left(i \sqrt{\frac{2 \Delta_{1}}{i \mu-\Delta_{1}}}\right)}{\sqrt{i \tilde{\Delta}_{1}+\mu}}-\Delta_{1} \sqrt{i \Delta_{1}+\mu} \sqrt{\frac{i \mu-\Delta_{1}}{i \mu+\Delta_{1}}}\left[\frac{i \mu+\Delta_{1}}{i \mu-\Delta_{1}} E\left(\sqrt{\frac{i \mu-\Delta_{1}}{i \mu+\Delta_{1}}}\right)-\sqrt{\frac{i \mu+\Delta_{1}}{i \mu-\Delta_{1}}} E\left(\sqrt{\frac{i \mu+\Delta_{1}}{i \mu-\tilde{\Delta}_{1}}}\right)\right. \\
& \left.-\frac{2 \Delta_{1}}{i \mu-\Delta_{1}} K\left(\sqrt{\frac{i \mu-\Delta_{1}}{i \mu+\Delta_{1}}}\right)\right]=(\rho-1) \frac{k_{0}}{k_{\mathrm{Ft}}} \Delta_{1}-\frac{\pi}{2 k_{\mathrm{F} 2} a_{22}} \frac{k_{\mathrm{F} 2}}{k_{\mathrm{Ft}}} \Delta_{1}-\frac{3}{4} \rho \tilde{U}_{12} \Delta_{2}, \\
& \frac{i \Delta_{2} K\left(i \sqrt{\frac{2 \Delta_{2}}{i\left(\mu-E_{\mathrm{g}}\right)-\Delta_{2}}}\right)}{\sqrt{i \Delta_{1}+\mu-E_{\mathrm{g}}}}-\Delta_{2} \sqrt{i \Delta_{2}+\mu-E_{\mathrm{g}}} \sqrt{\frac{i\left(\mu-E_{\mathrm{g}}\right)-\Delta_{2}}{i\left(\mu-E_{\mathrm{g}}\right)+\Delta_{2}}}\left[\frac{i\left(\mu-E_{\mathrm{g}}\right)+\Delta_{2}}{i\left(\mu-E_{\mathrm{g}}\right)-\Delta_{2}} E\left(\sqrt{\frac{i\left(\mu-E_{\mathrm{g}}\right)-\Delta_{2}}{i\left(\mu-E_{\mathrm{g}}\right)+\Delta_{2}}}\right)\right. \\
& \left.-\sqrt{\frac{i\left(\mu-E_{\mathrm{g}}\right)+\Delta_{2}}{i\left(\mu-E_{\mathrm{g}}\right)-\Delta_{2}}} E\left(\sqrt{\frac{i\left(\mu-E_{\mathrm{g}}\right)+\Delta_{2}}{i\left(\mu-E_{\mathrm{g}}\right)-\Delta_{2}}}\right)-\frac{2 \Delta_{2}}{i\left(\mu-E_{\mathrm{g}}\right)-\Delta_{2}} K\left(\sqrt{\frac{i\left(\mu-E_{\mathrm{g}}\right)-\Delta_{2}}{i\left(\mu-E_{\mathrm{g}}\right)+\Delta_{2}}}\right)\right] \\
& =-\frac{\pi}{2 k_{\mathrm{F} 2 a_{22}} \frac{k_{\mathrm{F} 2}}{k_{\mathrm{Ft}}} \Delta_{2}-\frac{3}{4} \rho \tilde{U}_{21} \Delta_{1} .}
\end{aligned}
$$

One can see that for Eq. (B13) of the system there is no dependence on the momentum cutoff $k_{0}$, while for Eq. (B12) this dependence is present, except for the specific case of coinciding intraband coupling strengths when $\rho=1$.

If one follows instead the strategy for the description of BCS-BEC properties with fixed scattering lengths in the two bands, then after similar analytical calculations, the equations for the energy gaps at zero temperature transform to

$$
\begin{aligned}
& \frac{i \Delta_{1} K\left(i \sqrt{\frac{2 \Delta_{1}}{i \mu-\Delta_{1}}}\right)}{\sqrt{i \Delta_{1}+\mu}}-\Delta_{1} \sqrt{i \Delta_{1}+\mu} \sqrt{\frac{i \mu-\Delta_{1}}{i \mu+\Delta_{1}}}\left[\frac{i \mu+\Delta_{1}}{i \mu-\Delta_{1}} E\left(\sqrt{\frac{i \mu-\Delta_{1}}{i \mu+\Delta_{1}}}\right)-\sqrt{\frac{i \mu+\Delta_{1}}{i \mu-\Delta_{1}}} E\left(\sqrt{\frac{i \mu+\Delta_{1}}{i \mu-\Delta_{1}}}\right)\right. \\
& \left.-\frac{2 \Delta_{1}}{i \mu-\Delta_{1}} K\left(\sqrt{\frac{i \mu-\Delta_{1}}{i \mu+\Delta_{1}}}\right)\right]=-\frac{\pi}{2 k_{\mathrm{F} 1} a_{11}} \frac{k_{\mathrm{F} 1}}{k_{\mathrm{Ft}}} \Delta_{1}-\frac{3}{4} \tilde{U}_{12} \Delta_{2}, \\
& \frac{i \Delta_{2} K\left(i \sqrt{\frac{2 \Delta_{2}}{i\left(\mu-E_{\mathrm{g}}\right)-\Delta_{2}}}\right)}{\sqrt{i \Delta_{1}+\mu-E_{\mathrm{g}}}}-\Delta_{2} \sqrt{i \Delta_{2}+\mu-E_{\mathrm{g}}} \sqrt{\frac{i\left(\mu-E_{\mathrm{g}}\right)-\Delta_{2}}{i\left(\mu-E_{\mathrm{g}}\right)+\Delta_{2}}}\left[\frac{i\left(\mu-E_{\mathrm{g}}\right)+\Delta_{2}}{i\left(\mu-E_{\mathrm{g}}\right)-\Delta_{2}} E\left(\sqrt{\frac{i\left(\mu-E_{\mathrm{g}}\right)-\Delta_{2}}{i\left(\mu-E_{\mathrm{g}}\right)+\Delta_{2}}}\right)\right. \\
& \left.-\sqrt{\frac{i\left(\mu-E_{\mathrm{g}}\right)+\Delta_{2}}{i\left(\mu-E_{\mathrm{g}}\right)-\Delta_{2}}} E\left(\sqrt{\frac{i\left(\mu-E_{\mathrm{g}}\right)+\tilde{\Delta}_{2}}{i\left(\mu-E_{\mathrm{g}}\right)-\tilde{\Delta}_{2}}}\right)-\frac{2 \Delta_{2}}{i\left(\mu-E_{\mathrm{g}}\right)-\Delta_{2}} K\left(\sqrt{\frac{i\left(\mu-E_{\mathrm{g}}\right)-\Delta_{2}}{i\left(\mu-E_{\mathrm{g}}\right)+\Delta_{2}}}\right)\right] \\
& =-\frac{\pi}{2 k_{\mathrm{F} 2 a_{22}} \frac{k_{\mathrm{F} 2}}{k_{\mathrm{Ft}}} \Delta_{2}-\frac{3}{4}} \tilde{U}_{21} \Delta_{1},
\end{aligned}
$$

where there is no cutoff momentum dependence in the limit $k_{0} \gg k_{\mathrm{Ft}}$, contrary to what it happens with Eqs. (B12) and (B13). 
[1] H. Suhl, B. T. Matthias, and L. R. Walker, Bardeen-CooperSchrieffer Theory of Superconductivity in the Case of Overlapping Bands, Phys. Rev. Lett. 3, 552 (1959).

[2] V. A. Moskalenko, Superconductivity of metals taking into account overlapping of the energy bands, Fiz. Met. Metalloved. 8, 503 (1959)[Phys. Met. Metallogr. 8, 25 (1959)].

[3] S.-Z. Lin, Ground state, collective mode, phase soliton and vortex in multiband superconductors, J. Phys.: Condens. Matter 26, 493202 (2014).

[4] Y. Tanaka, Multicomponent superconductivity based on multiband superconductors, Supercond. Sci. Technol. 28, 034002 (2015).

[5] M. V. Milošević and A. Perali, Emergent phenomena in multicomponent superconductivity: An introduction to the focus issue, Supercond. Sci. Technol. 28, 060201 (2015).

[6] Y. Yerin and A. N. Omelyanchouk, Proximity and Josephson effects in microstructures based on multiband superconductors, Low Temp. Phys. 43, 1013 (2017).

[7] A. Perali, A. Bianconi, A. Lanzara, and N. L. Saini, The gap amplification at a shape resonance in a superlattice of quantum stripes: A mechanism for high Tc, Solid State Commun. 100, 181 (1996).

[8] A. Valletta, A. Bianconi, A. Perali, and N. L. Saini, Electronic and superconducting properties of a superlattice of quantum stripes at the atomic limit, Z. Phys. B 104, 707 (1997).

[9] P. Nozières and S. Schmitt-Rink, Bose condensation in an attractive fermion gas: From weak to strong coupling superconductivity, J. Low Temp. Phys. 59, 195 (1985).

[10] M. Randeria and E. Taylor, Crossover from bardeen-cooperschrieffer to bose-einstein condensation and the unitary fermi gas, Annu. Rev. Condens. Matter Phys. 5, 209 (2014).

[11] A. Perali, P. Pieri, G. C. Strinati, and C. Castellani, Pseudogap and spectral function from superconducting fluctuations to the bosonic limit, Phys. Rev. B 66, 024510 (2002).

[12] F. Palestini, A. Perali, P. Pieri, and G. C. Strinati, Dispersions, weights, and widths of the single-particle spectral function in the normal phase of a Fermi gas, Phys. Rev. B 85, 024517 (2012).

[13] F. Marsiglio, P. Pieri, A. Perali, F. Palestini, and G. C. Strinati, Pairing effects in the normal phase of a two-dimensional Fermi gas, Phys. Rev. B 91, 054509 (2015).

[14] A. Bianconi, Shape resonances in superstripes, Nat. Phys. 9, 536 (2013).

[15] D. Innocenti, N. Poccia, A. Ricci, A. Valletta, S. Caprara, A. Perali, and A. Bianconi, Resonant and cross-over phenomena in a multiband superconductor: Tuning the chemical potential near a band edge, Phys. Rev. B 82, 184528 (2010).

[16] A. Guidini and A. Perali, Band-edge BCS-BEC crossover in a two-band superconductor: Physical properties and detection parameters, Supercond. Sci. Technol. 27, 124002 (2014).

[17] K. Hashimoto, K. Cho, T. Shibauchi, S. Kasahara, Y. Mizukami, R. Katsumata, Y. Tsuruhara, T. Terashima, H. Ikeda, M. A. Tanatar, H. Kitano, N. Salovich, R. W. Giannetta, P. Walmsley, A. Carrington, R. Prozorov, and Y. Matsuda, A sharp peak of the zero-temperature penetration depth at optimal composition in $\mathrm{BaFe}_{2}\left(\mathrm{As}_{1-x} \mathrm{P}_{x}\right)_{2}$, Science 336, 1554 (2012).

[18] T. Shibauchi, A. Carrington, and Y. Matsuda, A quantum critical point lying beneath the superconducting dome in iron pnictides, Annu. Rev. Condens. Matter Phys. 5, 113 (2014).
[19] Y. Lubashevsky, E. Lahoud, K. Chashka, D. Podolsky, and A. Kanigel, Shallow pockets and very strong coupling superconductivity in $\mathrm{FeSe}_{x} \mathrm{Te}_{1-x}$, Nat. Phys. 8, 309 (2012).

[20] K. Okazaki, Y. Ito, Y. Ota, Y. Kotani, T. Shimojima, T. Kiss, S. Watanabe, C.-T. Chen, S. Niitaka, T. Hanaguri, H. Takagi, A. Chainani, and S. Shin, Superconductivity in an electron band just above the Fermi level: Possible route to BCS-BEC superconductivity, Sci. Rep. 4, 4109 (2014).

[21] S. Kasahara, T. Watashige, T. Hanaguri, Y. Kohsaka, T. Yamashita, Y. Shimoyama, Y. Mizukami, R. Endo, H. Ikeda, K. Aoyama, T. Terashima, S. Uji, T. Wolf, H. von Löhneysen, T. Shibauchi, and Y. Matsuda, Field-induced superconducting phase of FeSe in the BCS-BEC crossover, Proc. Natl. Acad. Sci. USA 111, 16309 (2014).

[22] S. Kasahara, T. Yamashita, A. Shi, R. Kobayashi, Y. Shimoyama, T. Watashige, K. Ishida, T. Terashima, T. Wolf, F. Hardy, C. Meingast, H. v. Löhneysen, A. Levchenko, T. Shibauchi, and Y. Matsuda, Giant superconducting fluctuations in the compensated semimetal FeSe at the BCS-BEC crossover, Nat. Commun. 7, 12843 (2016).

[23] S. Rinott, K. B. Chashka, A. Ribak, E. D. L. Rienks, A. TalebIbrahimi, P. Le Fevre, F. Bertran, M. Randeria, and A. Kanigel, Tuning across the BCS-BEC crossover in the multiband superconductor : An angle-resolved photoemission study, Sci. Adv. 3, 1602372 (2017).

[24] T. Hanaguri, S. Kasahara, J. Böker, I. Eremin, T. Shibauchi, and Y. Matsuda, Quantum Vortex Core and Missing Pseudogap in the Multiband BCS-BEC Crossover Superconductor FeSe, Phys. Rev. Lett. 122, 077001 (2019).

[25] M. Köhl, H. Moritz, T. Stöferle, K. Günter, and T. Esslinger, Fermionic Atoms in a Three Dimensional Optical Lattice: Observing Fermi Surfaces, Dynamics, and Interactions, Phys. Rev. Lett. 94, 080403 (2005).

[26] S. Ospelkaus, C. Ospelkaus, L. Humbert, K. Sengstock, and K. Bongs, Tuning of Heteronuclear Interactions in a Degenerate Fermi-Bose Mixture, Phys. Rev. Lett. 97, 120403 (2006).

[27] J. K. Chin, D. E. Miller, Y. Liu, C. Stan, W. Setiawan, C. Sanner, $\mathrm{K}$. Xu, and W. Ketterle, Evidence for superfluidity of ultracold fermions in an optical lattice, Nature (London) 443, 961 (2006).

[28] G. Pagano, M. Mancini, G. Cappellini, L. Livi, C. Sias, J. Catani, M. Inguscio, and L. Fallani, Strongly Interacting Gas of Two-Electron Fermions at an Orbital Feshbach Resonance, Phys. Rev. Lett. 115, 265301 (2015).

[29] M. Höfer, L. Riegger, F. Scazza, C. Hofrichter, D. R. Fernandes, M. M. Parish, J. Levinsen, I. Bloch, and S. Folling, Observation of an Orbital Interaction-Induced Feshbach Resonance in ${ }^{173} \mathrm{Yb}$, Phys. Rev. Lett. 115, 265302 (2015).

[30] R. Zhang, Y. Cheng, H. Zhai, and P. Zhang, Orbital Feshbach Resonance in Alkali-Earth Atoms, Phys. Rev. Lett. 115, 135301 (2015).

[31] M. Iskin and C. A. R. Sá de Melo, BCS-BEC crossover of a collective excitations in two-band superfluids, Phys. Rev. B 72, 024512 (2005).

[32] M. Iskin and C. A. R. Sá de Melo, Two-band superfluidity from the BCS to the BEC limit, Phys. Rev. B 74, 144517 (2006).

[33] M. Iskin and C. A. R. Sá de Melo, Evolution of two-band superfluidity from weak to strong coupling, J. Low Temp. Phys. 149, 29 (2007). 
[34] M. Iskin, Two-band superfluidity and intrinsic Josephson effect in alkaline-earth-metal Fermi gases across an orbital Feshbach resonance, Phys. Rev. A 94, 011604(R) (2016).

[35] D. Reyes, M. A. Continentino, F. Deus, and C. Thomas, Taillike regime and BCS-BEC crossover due to hybridization in a two-band superconductor, J. Phys.: Condens. Matter 30, 175601 (2018).

[36] S. Mondal, D. Inotani, and Y. Ohashi, Single-particle Excitations and Strong Coupling Effects in the BCS-BEC Crossover Regime of a Rare-Earth Fermi Gas with an Orbital Feshbach Resonance, J. Phys. Soc. Jpn. 87, 084302 (2018).

[37] A. V. Chubukov, I. Eremin, and D. V. Efremov, Superconductivity vs bound state formation in a two-band superconductor with small Fermi energy - applications to Fe-pnictides/chalcogenides and doped $\mathrm{SrTiO}_{3}$, Phys. Rev. B 93, 174516 (2016).

[38] S. Wolf, A. Vagov, A. A. Shanenko, V. M. Axt, A. Perali, and J. A. Aguiar, BCS-BEC crossover induced by a shallow band: Pushing standard superconductivity types apart, Phys. Rev. B 95, 094521 (2017).

[39] L. Salasnich, A. A. Shanenko, A. Vagov, J. A. Aguiar, and A. Perali, Screening of pair fluctuations in superconductors with coupled shallow and deep bands: A route to higher temperature superconductivity, Phys. Rev. B 100, 064510 (2019).

[40] H. Tajima, Y. Yerin, A. Perali, and P. Pieri, Enhanced critical temperature, pairing fluctuation effects, and BCS-BEC crossover in a two-band Fermi gas, Phys. Rev. B 99, 180503(R) (2019).

[41] Y. S. Yerin and A. N. Omelyanchouk, Coherent current states in a two-band superconductor, Low Temp. Phys. 33, 401 (2007).

[42] F. Palestini and G. C. Strinati, Temperature dependence of the pair coherence and healing lengths for a fermionic superfluid throughout the BCS-BEC crossover, Phys. Rev. B 89, 224508 (2014).

[43] M. Holland, S. J. J. M. F. Kokkelmans, M. L. Chiofalo, and R. Walser, Resonance Superfluidity in A Quantum Degenerate Fermi Gas, Phys. Rev. Lett. 87, 120406 (2001).
[44] Y. Ohashi and A. Griffin, BCS-BEC Crossover in A Gas of Fermi Atoms with a Feshbach Resonance, Phys. Rev. Lett. 89, 130402 (2002).

[45] G. M. Falco and H. T. C. Stoof, Crossover Temperature of Bose-Einstein Condensation in An Atomic Fermi Gas, Phys. Rev. Lett. 92, 130401 (2004).

[46] G. Singh, A. Jouan, G. Herranz, M. Scigaj, F. Sánchez, L. Benfatto, S. Caprara, M. Grilli, G. Saiz, F. Couëdo, C. FeuilletPalma, J. Lesueur, and N. Bergeal, Gap suppression at a Lifshitz transition in a multi-condensate superconductor, Nat. Mater. 18, 948 (2019).

[47] R. M. Fernandes, J. T. Haraldsen, P. Wolfle, and A. V. Balatsky, Two-band superconductivity in doped $\mathrm{SrTiO}_{3}$ films and interfaces, Phys. Rev. B 87, 014510 (2013).

[48] F. Pistolesi and G. C. Strinati, Evolution from BCS superconductivity to Bose condensation: Role of the parameter $k_{F} \xi_{F}$, Phys. Rev. B 49, 6356 (1994).

[49] N. Andrenacci, A. Perali, P. Pieri, and G. C. Strinati, Densityinduced BCS to Bose-Einstein crossover, Phys. Rev. B 60, 12410 (1999).

[50] L. Komendová, Yajiang Chen, A. A. Shanenko, M. V. Milošević, and F. M. Peeters, Two-Band Superconductors: Hidden Criticality Deep in the Superconducting State, Phys. Rev. Lett. 108, 207002 (2012).

[51] M. Silaev and E. Babaev, Microscopic theory of type-1.5 superconductivity in multiband systems, Phys. Rev. B 84, 094515 (2011).

[52] M. Silaev and E. Babaev, Microscopic derivation of twocomponent Ginzburg-Landau model and conditions of its applicability in two-band systems, Phys. Rev. B 85, 134514 (2012).

[53] S. N. Klimin, J. Tempere, G. Lombardi, and J. T. Devreese, Finite temperature effective field theory and two-band superfluidity in Fermi gases, Eur. Phys. J. B 88, 122 (2015).

[54] A. Gurevich, Enhancement of the upper critical field by nonmagnetic impurities in dirty two-gap superconductors, Phys. Rev. B 67, 184515 (2003). 